\title{
Variations in Ocean Surface Temperature due to Near-Surface Flow: Straining the Cool Skin Layer
}

\author{
ANDREW J. WELLS \\ Institute of Theoretical Geophysics, Department of Applied Mathematics and Theoretical Physics, \\ University of Cambridge, Cambridge, United Kingdom, and Department of Geology and Geophysics, \\ Yale University, New Haven, Connecticut \\ Claudia Cenedese And J. ThOMAs FARraR
}

Physical Oceanography Department, Woods Hole Oceanographic Institution, Woods Hole, Massachusetts

CHRISTOPHER J. ZAPPA

Lamont-Doherty Earth Observatory, Columbia University, New York, New York

(Manuscript received 16 January 2008, in final form 12 May 2009)

\begin{abstract}
The aqueous thermal boundary layer near to the ocean surface, or skin layer, has thickness $O(1 \mathrm{~mm})$ and plays an important role in controlling the exchange of heat between the atmosphere and the ocean. Theoretical arguments and experimental measurements are used to investigate the dynamics of the skin layer under the influence of an upwelling flow, which is imposed in addition to free convection below a cooled water surface. Previous theories of straining flow in the skin layer are considered and a simple extension of a surface straining model is posed to describe the combination of turbulence and an upwelling flow. An additional theory is also proposed, conceptually based on the buoyancy-driven instability of a laminar straining flow cooled from above. In all three theories considered two distinct regimes are observed for different values of the Péclet number, which characterizes the ratio of advection to diffusion within the skin layer. For large Péclet numbers, the upwelling flow dominates and increases the free surface temperature, or skin temperature, to follow the scaling expected for a laminar straining flow. For small Péclet numbers, it is shown that any flow that is steady or varies over long time scales produces only a small change in skin temperature by direct straining of the skin layer. Experimental measurements demonstrate that a strong upwelling flow increases the skin temperature and suggest that the mean change in skin temperature with Péclet number is consistent with the theoretical trends for large Péclet number flow. However, all of the models considered consistently underpredict the measured skin temperature, both with and without an upwelling flow, possibly a result of surfactant effects not included in the models.
\end{abstract}

\section{Introduction}

The temperature of the air-sea interface, or skin temperature, can significantly influence the transfer of heat, moisture, and gases between the ocean and atmosphere. Recent studies have used infrared (IR) imagery to estimate the temperature of the upper 10-100 $\mu \mathrm{m}$ below the air-sea interface, revealing temperature variations on hor-

Corresponding author address: Andrew Wells, Department of Geology and Geophysics, Yale University, P.O. Box 208109, New Haven, CT 06520-8109.

E-mail: andrew.j.wells@yale.edu izontal scales of centimeters to kilometers (Marmorino and Smith 2005; Jessup and Hesany 1996; Zappa et al. 2004; Farrar et al. 2007). An interesting example are temperature signals of the order $0.2^{\circ} \mathrm{C}$ that appear to be associated with oceanic internal gravity waves (Marmorino et al. 2004; Zappa and Jessup 2005; Farrar et al. 2007), which suggest that subsurface flows can generate substantial modification of the skin temperature.

There are several ways in which a subsurface velocity could modulate the skin temperature. The velocity signals could modify the temperature profiles by acting directly within the skin layer, the thin diffusive thermal boundary layer of approximately 1-mm depth immediately below 
the ocean surface. Alternatively, velocity signals may modify the skin temperature by indirect mechanisms, such as modulating the level of subsurface turbulence (Walsh et al. 1998; Farrar et al. 2007), modifying local surfactant concentration (Marmorino et al. 2008), or modifying surface wave properties to generate an additional flow. We focus on the direct influence of a velocity signal within the skin layer.

The skin temperature is often about $0.2^{\circ} \mathrm{C}$ cooler than the water immediately below it as a result of heat loss to the atmosphere via latent, sensible, and longwave radiative heat fluxes (the difference is sometimes as large as $0.5^{\circ} \mathrm{C}$ under conditions of strong cooling). The processes occurring within the skin layer lie at too small a scale to be resolved in large-scale climate models or in most field observations. The influence of the thermal boundary layer on skin temperature must therefore be parameterized, and hence a detailed understanding of the unresolved physics is desirable (Fairall et al. 1996).

Since the early measurements of Woodcock and Stommel (1947) using a mercury thermometer, there have been numerous field, laboratory, theoretical, and numerical studies examining processes that modify the skin layer. For low wind speeds, a free convective boundary layer develops (Katsaros et al. 1977). This boundary layer thins in the presence of strong winds because of additional shear-generated turbulence (see, e.g., Saunders 1967; Castro et al. 2003). The skin layer can also be modified by swell waves (Jessup and Hesany 1996), white-capping (Jessup et al. 1997), microbreaking waves (Zappa et al. 2004), or surfactants (Jarvis 1962; Saylor et al. 2000; Handler et al. 2003). The dynamically similar problem of air-sea gas transfer has also received considerable attention [see Jähne and Haußecker (1998) for a review].

Osborne (1965) presented a theory to describe the modulation of the skin temperature by waves and other flows that generate a straining flow in the skin layer. Surface straining models have also been used to describe the heat and gas transfer due to subsurface turbulence, by parameterizing the straining flow generated by turbulent eddies (e.g., Fortescue and Pearson 1967; McKenna and McGillis 2004; Turney et al. 2005). A surface straining model was also applied by Csanady (1990) to predict the gas transfer due to high vorticity wavelets. Experimental studies have mostly focused on measuring transfer properties averaged over large spatial regions; very few have directly examined the influence of a straining flow on the skin layer. Ewing and McAlister (1960) demonstrated that a turbulent jet directed toward the interface can modify the skin temperature, and Leighton et al. (2003) showed that direct numerical simulations of free convection were well described by a surface straining model. However, there has been no quantitative experimental evaluation of the direct influence of a straining flow on the skin temperature. In this paper we consider whether straining models accurately predict the dynamics of the skin layer for a known straining flow.

We consider the fundamental problem of the response of the skin layer to straining flow. This is relevant as a simplified description of the influence of a large-scale upwelling flow on skin temperature. In addition, the straining flows generated by upwelling form a building block in surface straining models of turbulent heat and gas transfer, arising whenever circulation is generated on a scale larger than the thermal boundary layer thickness (such as the flow generated by turbulent eddies). Hence, this fundamental problem may also provide insight into the combined influence of multiple sources of turbulence on the skin layer, which could be considered as a superposition of an additional straining flow on an initial turbulent state. We consider a range of theoretical arguments to predict the strength of flow required for an appreciable change in skin temperature via direct straining of the skin layer. The theoretical predictions are then compared to laboratory measurements of the response of the skin layer to a steady upwelling flow, focusing primarily on the strong flows that are predicted to yield significant and observable changes in skin temperature.

We begin in section 2 by reviewing previous models of the skin layer used to describe the influence of subsurface turbulence on skin temperature and the modifications arising when an additional upwelling flow is superimposed (Osborne 1965). We develop a new linear extension of a surface straining model to describe the combination of turbulence and an additional upwelling flow and show that the model predicts similar skin temperatures to the Osborne (1965) theory despite the difference in physical assumptions required by the two models. A nondimensional formulation allows us to identify distinct dynamical regimes for weak and strong upwelling flows, and we show that a strong upwelling flow is required to induce a significant change in the skin temperature. We use both models to derive timedependent solutions for flows with weak imposed advection that varies over long-time scales, and we show that the resulting behavior is quasi steady at leading order. This extends a result of Osborne (1965), for sinusoidally varying flow, to flows with a general form of time dependence. An additional model is also suggested, based on a bifurcation between the flow regimes, with strong upwelling flows acting to stabilize the flow and prevent free convection, but with buoyancy-driven instability allowing free convection to persist in the presence of weaker flow. 
In sections 3 and 4 , we describe the experimental procedure and results for measurements of the skin layer when upwelling flows of different strengths are added to an initial state of free convective turbulence. We observe a local increase in skin temperature above regions of upwelling flow. For strong upwelling flows, all three theories capture the mean trend of variation of the skin temperature with strain rate, but the distinction from free convection is less clear for weaker flows, with the data lending the strongest support to the theory based on instability. However, all theories underpredict the magnitude of the temperature difference between the free surface and the tank interior for all our experiments, possibly due to the influence of surface contamination in the experiments, an effect not represented in the models considered here.

In section 5 we conclude with a discussion of the implications of the theoretical results for ocean flows. An application of all three theories suggest that an appreciable change in skin temperature requires a large strain rate, such as might be generated by high vorticity wavelets (Okuda 1982) or microbreaking waves. However, the large-scale flows generated by internal gravity waves are unlikely to generate a significant change in skin temperature by direct straining of the skin layer, suggesting that a different physical mechanism is required to explain the field observations of internal wave signals in skin temperature.

\section{Theoretical background}

In many situations the surface of the ocean is cooled by a combination of radiative, evaporative, and sensible heat fluxes $Q_{\text {rad }}, Q_{\text {evap }}$ and $Q_{\text {sens }}$, respectively. If the ocean occupies $z \leq 0$, then the net heat flux $Q$ generates a temperature gradient $\partial T / \partial z$ that satisfies

$-\left.\rho c_{p} \kappa \frac{\partial T}{\partial z}\right|_{z=0}=Q=Q_{\mathrm{rad}}+Q_{\text {evap }}+Q_{\text {sens }}-Q_{\text {sol }}$,

where $\rho, c_{p}$, and $\kappa$ are the density, specific heat capacity, and thermal diffusivity of the ocean, respectively. The term $Q_{\text {sol }}$ represents the depth-absorbed solar radiation, with less than $10 \%$ of the total solar radiation being absorbed within the skin layer (Wick et al. 2005). Typically $Q \approx 200 \mathrm{~W} \mathrm{~m}^{-2}$ for a nighttime ocean-to-atmosphere heat flux (see, e.g., Wick et al. 1996). A positive $Q$ corresponds to a net heat flux from the ocean to the atmosphere in our notation. The cooling creates a layer of dense fluid at the surface, which for large $Q$ inevitably leads to free convective turbulence below the ocean surface. This can be supplemented by turbulence driven by wind shear or other mechanisms, including wave breaking.
There is no normal flow across the air-water interface, so turbulent fluid motion is suppressed in a narrow layer close to the interface where molecular diffusion is important. Significant temperature change can occur across this diffusive thermal boundary layer, so that we have a cool skin of lower temperature than the fluid in the bulk below. This skin layer can have a thickness of about $0.5 \mathrm{~cm}$ for free convective turbulence, reducing to about $0.1 \mathrm{~cm}$ when additional shear-driven turbulence is generated by wind.

Several types of theory have been proposed to describe the properties of this boundary layer, employing a variety of different underlying assumptions. One approach is to describe the skin layer properties using a model of an effective diffusive layer (Saunders 1967), with all the temperature variation confined to a diffusive layer of finite depth, as shown in Fig. 1a. This effective diffusive layer model for free convection will be described in more detail in section 2a. Alternatively, surface straining models (e.g., Fortescue and Pearson 1967; Csanady 1990) explicitly resolve vertical advection by parameterizing the strength of the turbulent flow and then calculate the resulting balance between advection and diffusion of heat (Fig. 1b). Surface straining models are reviewed in section $2 \mathrm{~b}$. A third approach uses surface renewal models (e.g., Howard 1966; Soloviev and Schlüssel 1994), which consider the diffusive growth of the boundary layer over time and parameterize a critical time scale for renewal. For free convection this will correspond to the boundary layer thickness reaching some critical value at which the boundary layer becomes unstable and breaks up. The statistically steady properties of the boundary layer can then be described by averaging the resulting profiles in time. However, it is not immediately clear how to extend such a surface renewal model for the addition of an imposed laminar upwelling flow, so we do not consider this type of model in detail here.

In section $2 \mathrm{c}$ we consider how the effective diffusive layer model and surface straining model can be extended to describe the addition of an upwelling flow to free convective turbulence, appropriate to the laboratory experiments considered in sections 3 and 4 .

\section{a. Model of an effective diffusive layer}

Effective diffusive layer models describe the statistically steady properties of the skin layer by comparison with an idealized slab model, with all turbulence suppressed in a laminar layer $-\delta_{e} \leq z \leq 0$ lying above a turbulent interior $z<-\delta_{e}$ (Fig. 1a). The strength of the turbulent flow is parameterized by $\delta_{e}$, the effective depth of the laminar layer. The laminar layer is subject to boundary conditions 

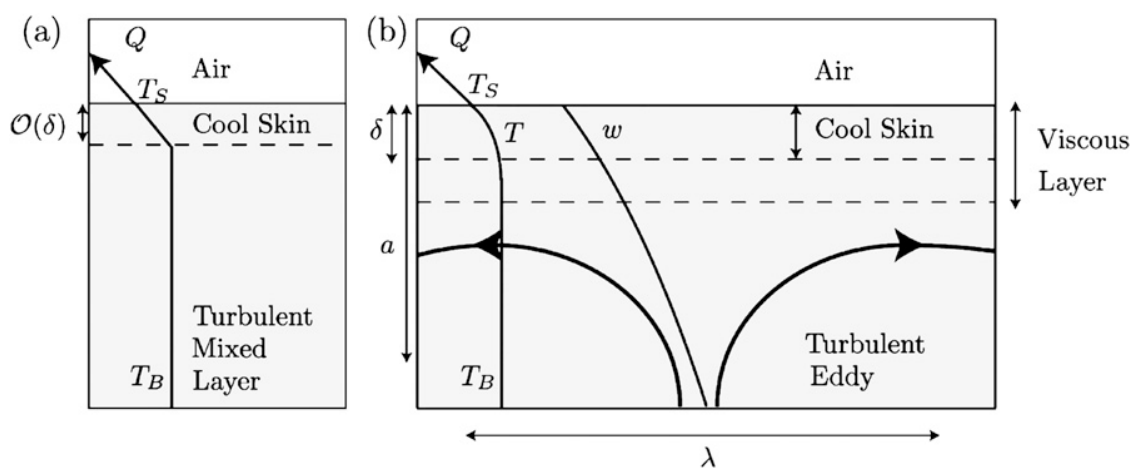

FIG. 1. Models of the surface thermal boundary layer of the ocean. (a) Effective diffusive layer model. A cold, laminar diffusive boundary layer of depth $O(\delta)$ overlies a turbulent wellmixed interior. This results in a skin temperature $T_{S}$ that is cooler than the mixed layer, which is at the uniform temperature $T_{B}$. (b) Surface straining model. Larger-scale flow features, such as turbulent eddies, act to generate a local straining flow in the boundary layer, with a vertical velocity $w$ that varies linearly with depth across the viscous boundary layer. The temperature profile $T(z)$ is then determined by a balance between the vertical advection and diffusion of heat in the thermal boundary layer above the region of upwelling flow. The thermal boundary layer has a depth of $O(\delta)$ that is much smaller than the vertical length scale $a$ and horizontal length scale $\lambda$ over which the velocity varies.

$$
\begin{aligned}
-\rho c_{p} \kappa \frac{\partial T}{\partial z} & =Q \quad \text { at } \quad z=0 \quad \text { and } \\
T & =T_{B} \quad \text { at } \quad z=-\delta_{e},
\end{aligned}
$$

corresponding to an imposed heat loss $Q$ to the atmosphere, and the well-mixed turbulent layer acting to maintain a uniform bulk temperature $T_{B}$ at the base of the skin layer. The temperature profile $T(z)$ in the laminar layer then determines the skin temperature $T_{S}$ at the free surface. In the absence of an imposed flow, the heat transfer is dominated by diffusion in this laminar layer, so that the temperature varies linearly with depth and yields a bulk-skin temperature difference

$$
T_{B}-T_{S}=\frac{Q \delta_{e}}{\rho c_{p} \kappa}
$$

Note that the effective diffusive layer is somewhat idealized and is only intended for prediction of the mean temperature change across the skin layer. Individual measurements will show variation about the mean state, and the model temperature profile will not necessarily correspond to those in the actual flow.

For free convection, Saunders (1967) suggested that the laminar layer thickness $\delta_{e}$ can be determined in terms of the heat flux $Q$ by applying the "four-thirds" heat transfer law for turbulent Rayleigh-Bénard convection. Scaling theories suggest that the temperature difference between the free surface and the interior should satisfy

$$
T_{B}-T_{S}=A^{-3 / 4}\left(\frac{Q}{\rho c_{p} \kappa}\right)^{3 / 4}\left(\frac{\kappa \nu}{\beta g}\right)^{1 / 4},
$$

where the constant of proportionality can be determined either from experiment, yielding $A=0.156$ for free convection below a cooled free surface (Katsaros et al. 1977), or from direct numerical simulations that yield $A=0.29$ (Leighton et al. 2003). The difference between these values will be discussed in section 4 . The acceleration due to gravity is denoted as $g$ here, $\beta$ is the thermal expansion coefficient, and $\nu$ is the kinematic viscosity of the fluid. Combining (3) with (4), we determine an effective diffusive layer thickness

$$
\delta_{e}=A^{-3 / 4}\left(\frac{Q}{\rho c_{p} \kappa}\right)^{-1 / 4}\left(\frac{\kappa \nu}{\beta g}\right)^{1 / 4}
$$

for free convection. The scale (5) is also consistent with the laminar layer having a thickness controlled by a buoyancy-driven instability, maintaining a constant Rayleigh number $\operatorname{Ra}_{\delta}=\beta g Q \delta_{e}^{4} / \rho c_{p} \kappa^{2} \nu=A^{-3} \sim 260$ or 40 for the different values of $A$, following the principles described by surface renewal theories (Howard 1966). As a rough comparison, both values are of similar order of magnitude as the critical value $\mathrm{Ra}_{\delta}=120$ obtained for Rayleigh-Bénard convection with a constant heat flux imposed at two free slip boundaries separated by a distance $\delta_{e}$ (Chapman and Proctor 1980).

\section{b. Surface straining models}

If flow is generated on a scale that is large compared to the thickness of the thermal boundary layer, then regions of upward flow can be approximated by a straining flow on the scale of the thermal boundary layer. This 
representation is used in surface straining models to describe turbulent flows, by resolving a turbulent eddy close to the free surface (such as the vortex pairs considered by Smith et al. 2001). However, the local straining flow is likely to be a general feature of many flows, such as the large-scale flow generated by internal waves, or the flow of the jet in the laboratory experiments described in section 3 . We review the ideas behind the straining flow approximation in a general context and then apply them to describe free convective turbulence in a surface straining model. The underlying principles of the straining flow approximation will also prove useful in section $2 \mathrm{c}$ when describing the influence of a superimposed upwelling flow.

Conservation of heat within the fluid can be described by the advection-diffusion equation

$$
\frac{\partial T}{\partial t}+\mathbf{u} \cdot \nabla T=\kappa \nabla^{2} T
$$

where $\mathbf{u}$ is the velocity of the fluid, which can be generated either by the imposed flow or by turbulence below the free surface. Note that we intend to explicitly resolve the upwelling flow due to turbulent eddies, and so we have not taken the Reynolds average here so that any "eddy flux" is still incorporated in the advection term. The flow is generated deep in the interior and has velocity $\mathbf{u}=(u, v, w)$ with characteristic interior velocity scales given by $\mathbf{u} \sim(\mathcal{U}, \mathcal{V}, \mathcal{W})$ varying over a vertical length scale $a$ and a horizontal wavelength $\lambda$, as illustrated in Fig. 1b. We assume that the thermal boundary layer has a thickness of characteristic scale $\delta$ much smaller than the scale of the imposed flow $(\delta \ll a, \lambda)$. This condition should be satisfied for any turbulent eddies or other turbulent flow structures that necessarily vary on length scales $a$ and $\lambda$ at least as large as the characteristic viscous boundary layer thickness. For fluid of large Prandtl number, $\operatorname{Pr}=\nu / \kappa>1$, we expect the thermal boundary layer to be thinner than the viscous boundary layer, so that $\delta$ is much smaller than both $a$ and $\lambda$. The condition is also strongly satisfied for flows generated deep in the ocean mixed layer that will be considered later (e.g., typically internal waves have $\lambda \approx 10-100 \mathrm{~m}, a \approx 1-10 \mathrm{~m}$, and $\delta<0.5 \mathrm{~cm}$ ).

There is no normal flow relative to the interface and so the vertical velocity $w=0$ at $z=0$, working in a reference frame where the air-water interface is fixed. The vertical velocity varies on the large length scale $a$, hence we can approximate

$$
w=\left.z \frac{\partial w}{\partial z}\right|_{z=0}+O\left(\mathcal{W} \frac{\delta^{2}}{a^{2}}\right), \quad-\delta \leq z \leq 0,
$$

within the narrow thermal boundary layer. Continuity requires that local upwelling is accompanied by a hori- zontally diverging flow, so that there is a local straining flow

$$
\begin{aligned}
& w=-E z, \quad u=E_{1} x, \quad v=E_{2} y, \quad \text { and } \\
& E=E_{1}+E_{2},
\end{aligned}
$$

where $E=-\partial w / \partial z$ is the vertical strain rate at $z=0$ and $(x, y)$ are local horizontal coordinates relative to the center of the divergence. The separation of length scales $\delta \ll \lambda$ implies that temperature variations satisfy $\partial_{x}$, $\partial_{y} \ll \partial_{z}$, thus horizontal diffusion is negligible compared to vertical diffusion across the boundary layer. Additionally, if $Q$ is independent of $x$ and $y$, we expect horizontal differences in temperature, with scale $\Delta \mathcal{T}_{H}$, to be small compared to the vertical bulk-skin temperature difference with scale $\Delta \mathcal{T}_{V}$. Within the skin layer of thickness $O(\delta),(8)$ yields velocity scalings $w \sim \mathcal{W} \delta / a$ and $u \sim \mathcal{W} \lambda / a$, thus the advection terms scale as

$$
\begin{aligned}
u \frac{\partial T}{\partial x} & \sim \frac{\mathcal{W} \lambda}{a} \frac{\Delta \mathcal{T}_{H}}{\lambda}, \quad w \frac{\partial T}{\partial z} \sim \frac{\mathcal{W} \delta}{a} \frac{\Delta \mathcal{T}_{H}}{\delta}, \quad \text { and } \\
\Delta \mathcal{T}_{H} & \ll \Delta \mathcal{T}_{V},
\end{aligned}
$$

and hence $\mathbf{u} \cdot \nabla T \approx w \partial_{z} T$. The heat equation (6) then simplifies to yield a possibly unsteady balance between vertical advection and vertical diffusion,

$$
\frac{\partial T}{\partial t}-E z \frac{\partial T}{\partial z}=\kappa \frac{\partial^{2} T}{\partial z^{2}} .
$$

This describes the influence of a straining flow in the skin layer, and different forms of the model are obtained by solving (10) with different boundary conditions or different specifications of the strain rate $E$.

The surface straining model describes turbulent heat transfer by parameterizing the strain rate $E$ to describe the turbulent flow (e.g., Turney et al. 2005). The boundary conditions are

$$
\begin{aligned}
-\rho c_{p} \kappa \frac{\partial T}{\partial z} & =Q \quad \text { at } \quad z=0 \quad \text { and } \\
T & \rightarrow T_{B} \quad \text { as } \quad \frac{z}{\delta} \rightarrow-\infty,
\end{aligned}
$$

corresponding to an imposed heat loss at the free surface and asymptotic matching to the bulk temperature as we move into the well-mixed region below the thermal boundary layer. The heat equation (10) has a steady solution,

$$
T(z)-T_{B}=\frac{Q}{\rho c_{p}} \sqrt{\frac{\pi}{2 E \kappa}} \operatorname{erfc}\left[-z \sqrt{\frac{E}{2 \kappa}}\right],
$$

so that the temperature variation occurs over a region with vertical scale $\sqrt{\kappa / E}$. The complementary error function and error function are defined by 


$$
\begin{aligned}
\operatorname{erfc}[x] & \equiv 1-\operatorname{erf}[x] \text { and } \\
\operatorname{erf}[x] & =\frac{2}{\sqrt{\pi}} \int_{0}^{x} \exp \left(-\xi^{2}\right) d \xi
\end{aligned}
$$

Note that if $Q$ is independent of $x$ and $y$, then (12) is also an exact solution of (6) with the horizontal advection terms retained. The scaling conditions (9) indicate when the assumption of constant $Q$ is reasonable. Evaluating (12) at $z=0$, we obtain the bulk-skin temperature difference

$$
T_{B}-T_{S}=\frac{Q}{\rho c_{p}} \sqrt{\frac{\pi}{2 \kappa E}} .
$$

Leighton et al. (2003) applied (12) and (14) to describe turbulent free convection due to the cooling of a water surface. They parameterized the strain rate $E=\gamma$, where $\gamma$ is determined on dimensional grounds in terms of the turbulent dissipation rate and its balance with the energy input from buoyancy,

$$
\nu \gamma^{2} \sim 2 \nu \frac{\partial u_{i}}{\partial x_{j}} \frac{\partial u_{i}}{\partial x_{j}} \sim \beta g \frac{Q}{\rho c_{p}} .
$$

The resulting prediction of bulk-skin temperature difference is identical to (4) if the constant of proportionality in (15) is chosen to satisfy

$$
\gamma=A^{3 / 2} \frac{\pi}{2}\left(\frac{\beta g Q}{\rho c_{p} \nu}\right)^{1 / 2} .
$$

The scalings give good collapse of the temperature profiles calculated in direct numerical simulations, using a value $A=0.29$, which is slightly larger than the value measured in the experiments of Katsaros et al. (1977).

\section{c. Influence of an additional upwelling flow}

Both the effective diffusive layer model and the surface straining model can be extended to describe the influence of an additional imposed upwelling flow, with characteristic strain rate $\alpha$, added as a perturbation to the initial free convective boundary layer.

\section{1) OSBORNE'S MODEL OF STRAINING IN AN EFFECTIVE DIFFUSIVE LAYER}

Osborne (1965) described the influence of the flow generated by internal and surface gravity waves on skin temperature, by applying an effective diffusive layer model in the style of section 2a to describe turbulent convection and then adding the straining flow due to waves as an additional perturbation. The temperature variation within the effective diffusive layer is described by (10) with $E=\alpha$ describing the strain induced by the waves. The boundary conditions are given by (2), so that information about the strength of the initial turbulent flow is carried in the effective skin layer thickness $\delta_{e}$ [which is given by (5) in the case of free convective turbulence]. This yields a bulk-skin temperature difference

$$
T_{B}-T_{S}=\frac{Q}{\rho c_{p}} \sqrt{\frac{\pi}{2 \kappa \alpha}} \operatorname{erf}\left[\delta_{e} \sqrt{\frac{\alpha}{2 \kappa}}\right] .
$$

Osborne (1965) assumes that either $\delta_{e}$ is constant or alternatively treats the case in which the fluid at $z=\delta_{e}$ is advected as a material surface by a sinusoidally varying vertical flow. It is not clear a priori if these assumptions are valid. Note that when there is no imposed flow $(\alpha=$ $0)$, (17) recovers the relation (3) for the effective diffusive layer model. For large strain rates with $\alpha \delta_{e}^{2} \kappa \gg 1$, Osborne showed that the temperature difference approaches (14) with $E \sim \alpha$, consistent with the limit for a laminar straining flow. ${ }^{1}$

\section{2) Surface STRAining MOdEL WITH AN ADDITIONAL IMPOSED FLOW}

In this section we propose two possible models for how the surface straining model of section $2 \mathrm{~b}$ might be extended to describe an imposed upwelling flow added to free convective turbulence. The combined flow will be described by some overall strain rate $E(\alpha)$ that depends on the imposed strain $\alpha$ and the strength of the free convective turbulence. We expect

$$
E(0)=\gamma \quad \text { and } \quad E \sim \alpha \quad \text { as } \quad \alpha / \gamma \rightarrow \infty .
$$

This gives the scaling of Leighton et al. (2003) for free convection with no imposed flow and recovers the limit of a simple straining flow when the flow due to free convection is relatively weak. A very simple approach is to assume the linear approximation

$$
E=\gamma+\alpha,
$$

corresponding to adding the components of velocity due to free convection and the imposed flow. This will be referred to as the linear surface straining model (LSSM) throughout the remainder of this work. This approach relies on there being no interaction between the free convection and the imposed flow, and the justification for this approximation is not clear.

An alternative approach is to consider the stability of a simple straining flow below a cooled free surface,

\footnotetext{
${ }^{1}$ Note that the corresponding equation in Osborne [1965, his Eq. (19)] contains a typographical error.
} 
TABLE 1. Comparison of the nondimensional form of the Osborne (1965) model and the linear surface straining model (LSSM) with $E=\gamma+\alpha$. The dimensionless bulk-skin temperature difference is characterized by $1 / \mathrm{Nu}=\rho c_{p} \kappa\left(T_{B}-T_{S}\right) / Q \delta_{e}$ and varies depending on the strength of the imposed flow with strain $\alpha=\alpha_{0} f(\omega t)$, which is described by a Péclet number Pe $=\alpha_{0} \delta_{e}^{2} / \kappa$. The Osborne (1965) and linear surface straining models agree for strong imposed flows $(\mathrm{Pe} \gg 1)$ and only show a small percentage difference when the imposed advection is weak $(\mathrm{Pe} \ll 1)$. The unsteady solutions $(\mathrm{T} 7)$ are derived in appendix $\mathrm{A}$.

\begin{tabular}{|c|c|c|c|}
\hline \multirow[t]{2}{*}{ Heat equation } & \multicolumn{2}{|c|}{$\operatorname{StPe} \frac{\partial \theta}{\partial \hat{t}}-g(\hat{t}) \hat{z} \frac{\partial \theta}{\partial \hat{z}}=\frac{\partial^{2} \theta}{\partial \hat{z}^{2}}$} & \multirow[t]{2}{*}{ (T1) } \\
\hline & (a) Osborne & (b) LSSM & \\
\hline \multirow{3}{*}{ Boundary conditions } & $g(\hat{t})=\operatorname{Pe} f(\hat{t})$ & $g(\hat{t})=\frac{\pi}{2}+\operatorname{Pe} f(\hat{t})$ & $(\mathrm{T} 2)$ \\
\hline & $\frac{\partial \theta}{\partial \hat{z}}=-1 \quad$ at $\quad \hat{z}=0$ & $\frac{\partial \theta}{\partial \hat{z}}=-1 \quad$ at $\quad \hat{z}=0$ & (T3) \\
\hline & $\theta=0 \quad$ at $\quad \hat{z}=-1$ & $\theta \rightarrow 0 \quad$ as $\quad \hat{z} \rightarrow-\infty$ & (T4) \\
\hline $\begin{array}{l}\text { Solution: } \\
\text { Full, steady }\end{array}$ & $\frac{1}{\mathrm{Nu}}=\sqrt{\frac{\pi}{2}} \frac{\operatorname{erf}\left[(\mathrm{Pe} / 2)^{1 / 2}\right]}{\mathrm{Pe}^{1 / 2}}$ & $\frac{1}{\mathrm{Nu}}=\left(1+\frac{2}{\pi} \mathrm{Pe}\right)^{-1 / 2}$ & (T5) \\
\hline Steady, $\mathrm{Pe} \gg 1$ & $\frac{1}{\mathrm{Nu}} \sim \sqrt{\frac{\pi}{2 \mathrm{Pe}}}$ & $\frac{1}{\mathrm{Nu}} \sim \sqrt{\frac{\pi}{2 \mathrm{Pe}}}$ & (T6) \\
\hline Unsteady, $\mathrm{Pe} \ll 1$ & $\frac{1}{\mathrm{Nu}}=1-\frac{\mathrm{Pe} f(\hat{t})}{6}+O\left(\mathrm{Pe}^{2}\right)$ & $\frac{1}{\mathrm{Nu}}=1-\frac{\mathrm{Pe} f(\hat{t})}{\pi}+O\left(\mathrm{Pe}^{2}\right)$ & (T7) \\
\hline
\end{tabular}

following a similar approach to Howard (1966). The temperature profile (12) can also be used to describe a pure laminar straining flow (with $E=\alpha$ ) and shows that the thermal boundary layer thickness scales as $\sqrt{\kappa / \alpha}$, with a stronger flow creating a thinner boundary layer. We can use this scale to create a characteristic local Rayleigh number,

$$
\mathrm{Ra}_{\alpha}=\frac{g \beta Q}{\rho c_{p} \kappa^{2} \nu}\left(\sqrt{\frac{\kappa}{\alpha}}\right)^{4} .
$$

For a large strain rate, the Rayleigh number is small and by analogy with Rayleigh-Bénard convection the flow is stable. As the strain rate $\alpha$ is reduced, the Rayleigh number increases until a critical value is exceeded, the flow becomes unstable, and we have the onset of convection. This will give

$$
E=\alpha \text { for } \alpha>\alpha_{c}
$$

when the strain stabilizes the flow, and the strain rate will then approach the value $E(0)=\gamma$ over the range $\alpha<\alpha_{c}$ where the flow is unstable and convection ensues. The exact form of dependence and the exact critical value of strain rate cannot be determined without further detailed analysis. However, as a first approximation, we might assume that the bifurcation occurs when the imposed strain matches the effective strain rate $\gamma$ given by (16) and used to describe free convection in the surface straining model. This yields $\alpha_{c}=\gamma$ and

$$
E=\gamma \text { for } \alpha<\gamma .
$$

\section{d. Nondimensional formulation}

It is useful to describe both the Osborne (1965) and linear surface straining models in nondimensional form. This allows us to characterize the influence of the imposed strain compared to the initial free convection and also allows for the investigation of the asymptotic structure of the boundary layer along with some aspects of time-dependent behavior. For generality we consider a possibly time-dependent imposed flow $\alpha=\alpha_{0} f(\omega t)$ varying over a time scale $1 / \omega$ (or with a frequency $\omega$ for periodic flows). We scale variables relative to the initial free convective state, setting $\hat{z}=z / \delta_{e}, \theta=\rho c_{p} \kappa\left(T-T_{B}\right) / Q \delta_{e}$, and $\hat{t}=\omega t$, where $\delta_{e} \equiv \sqrt{\pi \kappa / 2 \gamma}$ is the skin layer thickness for free convection given by (5) and $Q \delta_{e} / \rho c_{p} \kappa$ is the bulkskin temperature difference given by (4) from the fourthirds law for free convection. The nondimensional form of the heat equation is summarized in Table 1, along with the corresponding solutions from the Osborne (1965) model and the linear surface straining model. The dimensionless Péclet number

$$
\mathrm{Pe}=\frac{\alpha_{0} \delta_{e}^{2}}{\kappa}
$$

measures the importance of the imposed advection of heat relative to diffusion. Note that for our experiments, the initial skin layer depth $\delta_{e}$ is determined by free convection, so that the Péclet numbers will also indicate the importance of the imposed advection of heat relative to free convection in our case. The Strouhal number 


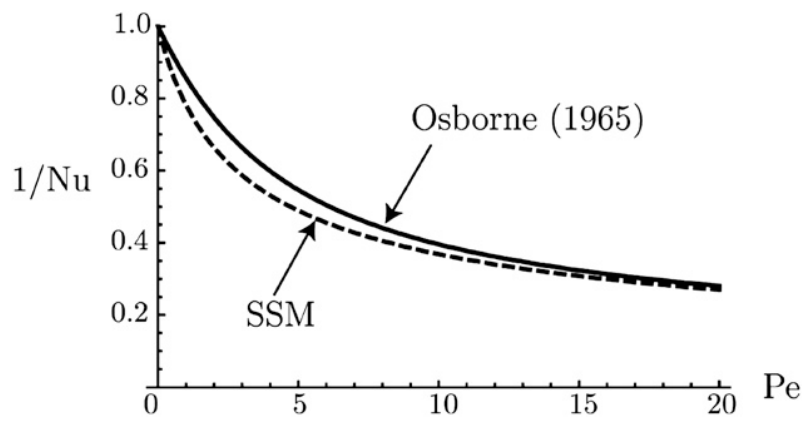

FIG. 2. Variation of the nondimensional bulk-skin temperature difference $1 / \mathrm{Nu}=\rho c_{p} \kappa\left(T_{B}-T_{s}\right) / Q \delta_{e}$ with Péclet number $\mathrm{Pe}=\alpha_{0} \delta_{e}^{2} / \kappa$ for a steady flow with $f(\hat{t})=1$. The solid curve shows the prediction (T5a) for the Osborne (1965) theory, and the dashed curve represents (T5b) for the LSSM.

$$
\mathrm{St}=\frac{\omega}{\alpha_{0}}
$$

is the dimensionless frequency (or dimensionless inverse time scale) of the imposed flow. The nondimensional bulk-skin temperature difference is described by an inverse Nusselt number

$$
\frac{1}{\mathrm{Nu}}=\frac{\rho c_{p} \kappa\left(T_{B}-T_{S}\right)}{Q \delta_{e}}
$$

which represents the ratio of the bulk-skin temperature difference in the presence of an imposed flow to that for free convection with $\alpha=0$.

Figure 2 compares the nondimensional bulk-skin temperature difference predicted by [(T5a); see Table 1] for the Osborne (1965) theory and by (T5b) for the linear surface straining model with $E=\gamma+\alpha$. Despite the difference in physical assumptions employed by the two theories, there is only a small difference between the predicted skin temperatures for all imposed upwelling flows (with $\mathrm{Pe}>0$ ), with a maximum percentage difference of approximately $13 \%$. The models agree exactly for $\mathrm{Pe}=0$ and $\mathrm{Pe} \rightarrow \infty$, and they effectively describe different methods of matching between these limits. We can consider the asymptotic behavior of each of the theories in two different cases.

For small Péclet numbers $(\mathrm{Pe} \ll 1)$, the advection due to the imposed flow is weak compared to diffusion across the skin layer, and the bulk-skin temperature difference is described by the expansions (T7a,b). In appendix A we show that $(\mathrm{T} 7 \mathrm{a}, \mathrm{b})$ are also the leading order solutions of the corresponding unsteady problem (T1)-(T4) with general time-dependent forcing $f(\hat{t})$, provided that StPe $\ll 1$, so that the time scale of the forcing $1 / \omega$ is slow compared to the characteristic diffusion time $\delta^{2} / \kappa$ across the thin skin layer. This implies that the temperature

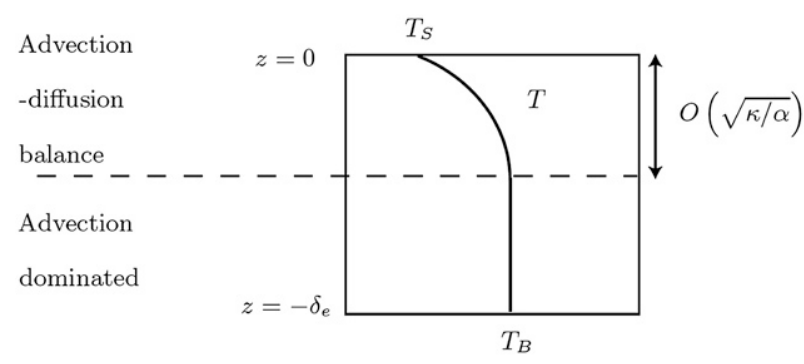

FIG. 3. Schematic illustration of the asymptotic structure of the thermal boundary layer in the Osborne (1965) model for large Péclet numbers. The lower region is dominated by advection of heat from the bulk and has constant temperature $T=T_{B}$. The temperature variation is confined to a boundary layer of depth $z=O(\sqrt{\kappa / \alpha})$ near to the surface, where there is a balance between advection and diffusion.

profile evolves in a quasi-steady fashion for many of the flows of long time scales that are observed in the ocean. This generalizes the corresponding derivation made by Osborne (1965) for sinusoidally varying flows. A consequence of the expansions $(\mathrm{T} 7 \mathrm{a}, \mathrm{b})$ is that the imposed advection generates only an $O(\mathrm{Pe})$ correction to the bulk-skin temperature difference, for both steady and low-frequency flows. A relatively large value of the Péclet number is required for skin layer straining to generate a significant percentage change in the bulk-skin temperature difference. This has important consequences for oceanic flows and will be discussed in more detail in section 5 .

For large Péclet numbers, the imposed advection dominates compared to the free convection, and the bulk-skin temperature difference is described by (T6) for both the Osborne (1965) theory and the linear surface straining model. The linear surface straining model yields a thermal boundary layer of thickness $z \approx O(\sqrt{\kappa / \alpha})$ for $\mathrm{Pe}=\pi \alpha / 2 \gamma \gg 1$, which is the same as the boundary layer thickness for a laminar straining flow with no convection. In appendix B we demonstrate that the temperature profiles $T(z)$ in the Osborne (1965) model describe similar behavior for Pe $\gg 1$. The modeled effective laminar layer divides into two distinct asymptotic regions, as illustrated in Fig. 3. A lower region is dominated by advection with the temperature equal to the bulk temperature $T_{B}$ at leading order. The temperature variation is confined to a near-surface layer of depth $\hat{z}=O\left(\mathrm{Pe}^{-1 / 2}\right)$, recovering $z \approx O(\sqrt{\kappa / \alpha})$ in dimensional form. Hence, the thermal boundary layer thickness is effectively reduced so as to maintain the advectiondiffusion balance across the reduced depth. This suggests that, for all models considered, a strong upwelling flow will dominate the effects of free convection.

In sections 3 and 4, we discuss an experimental investigation of the dynamics described by the theories 


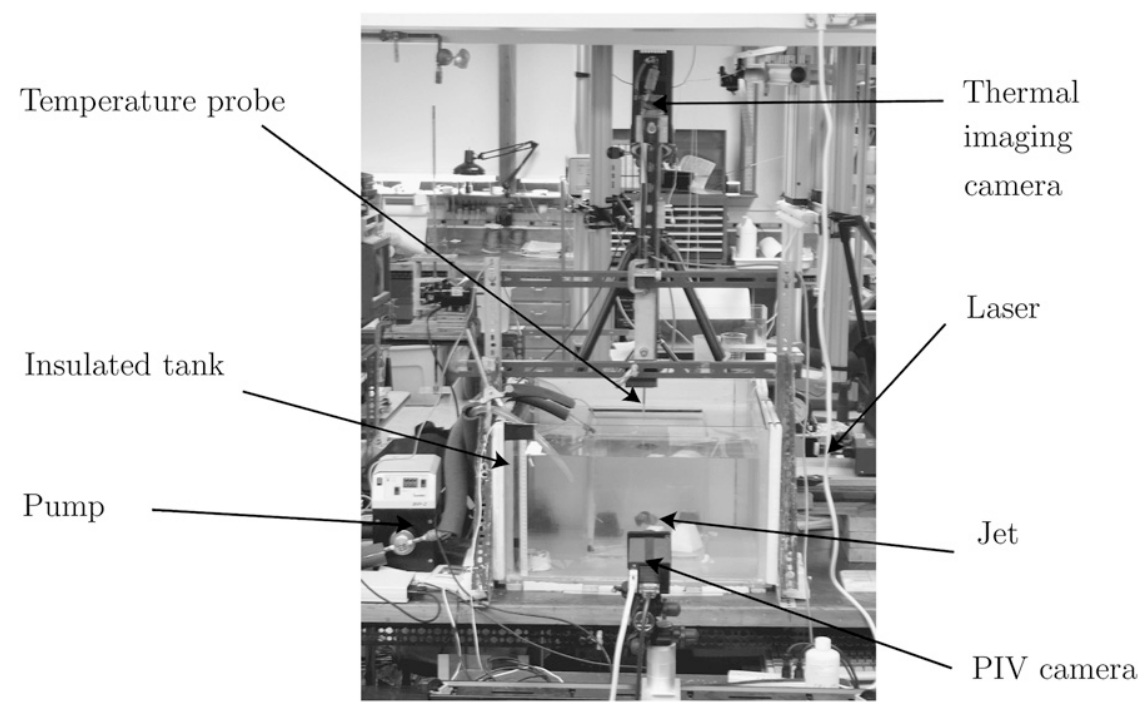

FIG. 4. The experimental apparatus.

discussed in this section, focusing on the case of a strong and steady flow that is expected to yield an appreciable change in skin temperature.

\section{Experimental procedure}

A laboratory experiment was used to investigate the variation of the cool skin effect with strain rate. The experimental setup is shown in Figs. 4 and 5. A tank of depth $30 \mathrm{~cm}$ and square cross section of width $60 \mathrm{~cm}$ was filled with tap water at temperature $T_{B}$ warmer than the ambient air temperature (which was maintained at approximately $18^{\circ} \mathrm{C}$ in the air-conditioned laboratory), so that a free convection thermal boundary layer forms below the free surface due to heat loss to the air. The water temperature was varied over $18.8^{\circ} \mathrm{C}<T_{B}<25.6^{\circ} \mathrm{C}$, to alter the temperature difference with the air and hence give different heat fluxes $Q$ across the free surface. A smaller inner tank of depth $30 \mathrm{~cm}$ and square cross section of width $30 \mathrm{~cm}$ was placed inside the outer tank, as shown in Fig. 5, to reduce secondary circulation in the measurement region, as discussed below. The sidewalls and base of the outer tank are insulated (along with all pipes connected to the pump), so that the dominant heat loss is across the air-water interface.

A laminar jet was created at the base of the inner tank by pumping fluid through a vertical nozzle covered by a sponge. The pump flow rate was varied over $0.12 \mathrm{~cm}^{3} \mathrm{~s}^{-1} \leq F \leq 1.14 \mathrm{~cm}^{3} \mathrm{~s}^{-1}$ to alter the input momentum flux and hence vary the strain rate close to the surface. The jet decelerates as it approaches the free surface, so that there is an axisymmetric straining flow close to the surface (indicated by the arrows in Fig. 5). The fluid then spreads radially, creating a surface divergence, before overflowing the edges of the inner tank into the outer tank where the fluid is returned to the pump via a

\section{SIDE VIEW:}

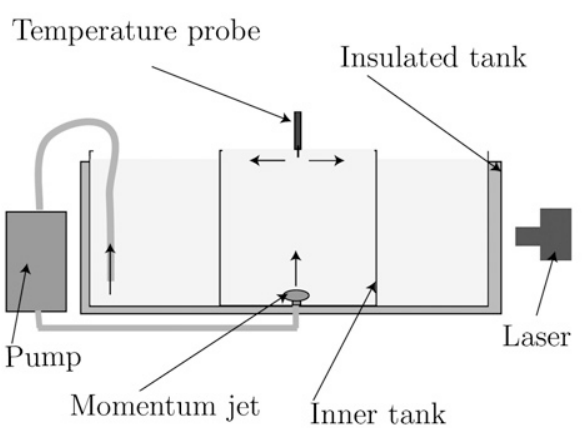

PLAN VIEW:

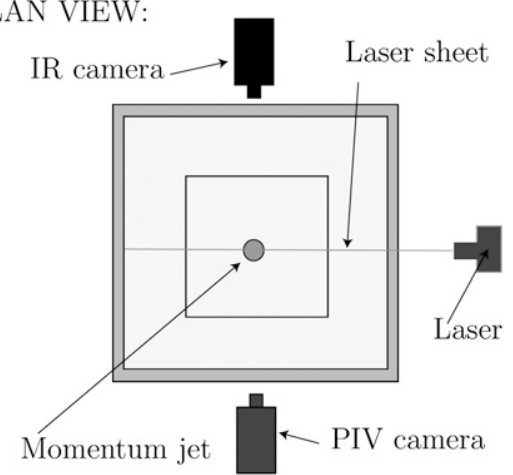

FIG. 5. Schematic diagram illustrating the experimental setup. Fluid is injected via a jet at the base of the inner tank to create a near-surface flow divergence, as indicated by the arrows. 

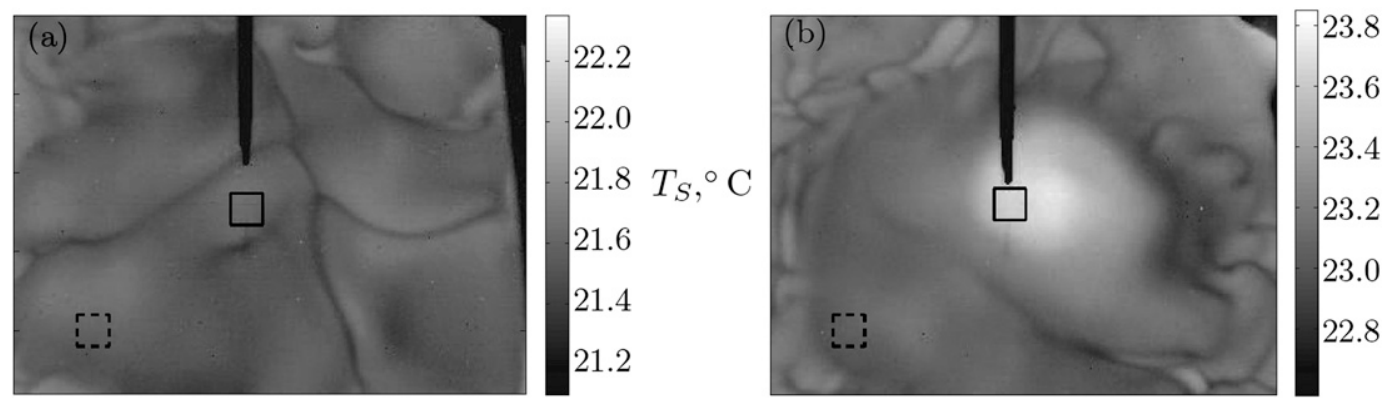

FIG. 6. Instantaneous images of the measured temperature $\left({ }^{\circ} \mathrm{C}\right)$ at the free surface, for (a) pure free convection and (b) an upwelling jet generating a strain rate $\alpha=0.158 \mathrm{~s}^{-1}$. The variation of grayscale in each image corresponds to variation in skin temperature, with lighter colored regions being warmer and darker regions being cooler. Each image shows a region of the surface $15 \mathrm{~cm} \times 12 \mathrm{~cm}$, with the $1 \mathrm{~cm} \times 1 \mathrm{~cm}$ central averaging region indicated by a black outline, and the far-field averaging region marked by a dashed line. The microscale temperature probe shows in black at the center of the image, shortly before it is lowered into the water. Convection roll structures are observed in the free convective case (a), with cold regions above local regions of downwelling. The relatively warm patch in the center of frame (b) corresponds to the thermal signature of the upwelling jet. Notice that the temperature scales differ for each image, with a different bulk temperature $T_{B}$ for each. The displayed patterns of skin temperature remain approximately constant over the 5-s sampling time of the IR camera, consistent with the sampling period being much shorter than the renewal time of a convection cell of $\delta^{2} / \kappa \approx 1000 \mathrm{~s}$.

sink. This overflow mechanism prevents the draining flow generated by the sink from influencing the upwelling flow in the inner tank.

The velocity field was measured using a LaVision particle image velocimetry (PIV) system. The tank was seeded with $10-\mu \mathrm{m}$ passive tracer particles, which were illuminated by a vertical laser sheet passing approximately through the center plane of the jet. The particle displacements between a pair of consecutive camera frames are then correlated to estimate the velocity components in the plane of illumination. An interval of $0.06 \mathrm{~s}$ between frames was found to give the best resolution of the flow. For each flow rate, a sequence of 155 image pairs was taken at a sampling rate of $4 \mathrm{~Hz}$. This sequence was time averaged, and a mean vertical velocity profile $w(z)$ was then calculated for each value of $z$ by taking a horizontal average of $w$ over a cross section of width $2 \mathrm{~cm}$ about the centerline of the jet.

The bulk temperature $T_{B}$ was measured with an accuracy of $0.1^{\circ} \mathrm{C}$ and a sensitivity of $0.02^{\circ} \mathrm{C}$ by a digital $\mathrm{HOBO}$ data logging thermometer placed at approximately $20-\mathrm{cm}$ depth in the outer tank. The bulk temperature value for each experiment was obtained from an average of 40 measurements over a 10-min period centered on the time of the experiment. To check the absolute calibration, the HOBO measurement was compared to a laboratory standard alcohol thermometer, with the mean difference of $0.07^{\circ} \mathrm{C}$ and a standard deviation of $0.09^{\circ} \mathrm{C}$, consistent with the quoted accuracy level.

The skin temperature $T_{S}$ was measured using a Cedip model Jade longwave IR thermal imaging camera, mounted $60 \mathrm{~cm}$ above the water surface with a viewing angle of $26^{\circ}$ to the vertical. This measures the temperature of the upper $10 \mu \mathrm{m}$ of water below the free surface. Before each day of measurements, the camera was calibrated against a known range of temperatures generated by a blackbody device, yielding an absolute accuracy of $0.1^{\circ} \mathrm{C}$ with a sensitivity of $0.02^{\circ} \mathrm{C}$. The skin temperature was determined by a spatial average over a $1 \mathrm{~cm}^{2}$ (441 pixel) square box centered on the point where the tip of the temperature probe pierces the interface and then an additional time average of 100 images taken over a 5-s period. Single images of the skin temperature variation are shown in Fig. 6, both with and without an imposed flow, with the measurement region marked by a black outline. Additional measurements of skin temperature were also obtained in the far field for comparison, by averaging over a second $1 \mathrm{~cm}^{2}$ box marked by a dashed outline in Fig. 6. On the first day of measurements, an independent check of the calibration between the thermal imaging camera and the digital thermometer was made by stirring the tank for a range of bulk temperatures (following Katsaros et al. 1977). For each bulk temperature, the tank was stirred vigorously to disrupt the skin layer and equilibrate the skin temperature close to the bulk temperature. The resulting maximum in skin temperature measured by the IR camera differed by an average of only $-0.03^{\circ} \mathrm{C}$ from the bulk temperature measured using the digital thermometer, suggesting that any bias between the instruments is small.

Independent temperature profiles were obtained to investigate the variation of temperature below the free surface. A PME microscale temperature probe (incorporating a Thermometrics FP07 thermistor) was lowered 
into the tank at a velocity of $0.3 \mathrm{~cm} \mathrm{~s}^{-1}$ to measure the variation of temperature with depth. The probe tip was aligned to pierce the interface close to the center of the upwelling jet. Temperature measurements were taken every $0.001 \mathrm{~cm}$ over the upper $5 \mathrm{~cm}$ of the tank interior, with a large number of points used to allow averaging to improve the accuracy. (Note that the time response of the thermistor is only $0.007 \mathrm{~s}$, therefore each point will be lagged by approximately $0.002 \mathrm{~cm}$ ). Downward sampling was used so that the probe tip could take measurements without obstructing the upwelling flow. Several measurements were also made with an upward moving U-shaped probe for the free convection case, and no appreciable differences were observed in the temperature profiles. This suggests that the vertical motion of the probe tip does not have a strong influence on the temperature measurement, as similar profiles are observed for both an upward and downward moving probe. The microscale temperature probe was calibrated directly against the HOBO digital thermometer before and after each session of measurements, with negligible drift observed. The analog output of the microscale temperature probe was subject to some contamination by electrical noise generated within the laboratory, which implies an accuracy of only $0.1^{\circ} \mathrm{C}$ for each individual measurement in the temperature profile, but a vertical running average of the measurements allows for improved sensitivity. Assuming uncorrelated errors from the laboratory noise, a 100-point running average reduces this error to significantly less than the $0.04^{\circ} \mathrm{C}$ sensitivity of the instrument.

The total heat flux across the air-water interface was estimated using calorimetry. If we assume negligible heat loss across the insulated sidewalls and the base of the tank, then most of the heat loss has to occur across the free surface. It is assumed that turbulent convection maintains the interior of the tank at a uniform temperature. The difference between the temperature profile measurements at 4-cm depth and the independent bulk temperature measurements at $20-\mathrm{cm}$ depth in the outer tank has a mean of $0.01^{\circ} \mathrm{C}$ and a standard deviation of $0.09^{\circ} \mathrm{C}$, suggesting that this is a reasonable approximation. The order 1-cm skin layer comprises less than $5 \%$ of the $30-\mathrm{cm}$ depth of the tank, and so we neglect the departure from the bulk temperature in this small region, assuming that the variation of the mean temperature $\bar{T}$ of the tank is approximately equal to the variation of $T_{B}$. Balancing the rate of change of heat within the tank with the heat flux across the free surface, we obtain

$$
\rho c_{p} \frac{d}{d t}(\mathcal{V} \bar{T})=-\mathcal{A} Q
$$

where $\mathcal{V}$ is the volume of water in the tank, and we assume $\bar{T} \approx T_{B}$ and that the water-to-air heat flux $Q$ is uniformly distributed across the free surface of area $\mathcal{A}$. When estimating $Q$, we neglect the change of the total volume of water in the tank with time. The measurements yield mean evaporation rates of less than $1 \mathrm{~cm} \mathrm{day}^{-1}$, resulting in an error of order $1 \mathrm{~W} \mathrm{~m}^{-2}$ from neglecting the term $\rho c_{p}\left(T_{B}-T_{S}\right)(1 / \mathcal{A}) d \mathcal{V} / d t$ due to loss of water by evaporation at the free surface. The assumption of uniform heat flux is a possible source of error over the jet. This is discussed in more detail in section $4 \mathrm{c}$, where we argue that the percentage change should be small because the variation in skin temperature is small compared to the temperature difference between the air and the water. The heat flux $Q$ was estimated using (26), and the bulk temperature measured with the HOBO digital thermometer. The time rate of change of $T_{B}$ at each time $t$ was calculated using a linear regression of temperature against time, applied on a time interval $(t-\Delta t, t+\Delta t)$. The range of the interval $2 \Delta t$ was increased until the $95 \%$ confidence interval of the predicted temperature gradient reached a minimum range, before the nonlinear variation of $T_{B}(t)$ became significant. Typical time intervals were $\Delta t=750 \mathrm{~s}$, and this provided a smooth variation of the heat flux in time.

The measurements mentioned earlier were taken for different strain rates (i.e., different flow rates for the pump-driven jet), and the bulk temperature was also varied to alter the heat flux $Q$. A complete list of experimental conditions is included in appendix C. The following procedure was adopted for each experiment. During a period of $5 \mathrm{~s}, 100$ thermal camera images were taken before a temperature-depth profile was taken with the temperature probe moving downward through the free surface. The bulk temperature was recorded continuously during several experiments. Particle image velocimetry measurements were taken for each momentum flux for a range of bulk temperatures. Initial measurements showed a negligible change in the mean value of the strain rate $\alpha$ with bulk temperature, suggesting that the dynamical effect of convection is relatively weak compared to that of the imposed flow of the jet. A single average value of $\alpha$ was therefore used for each pump flow rate in the subsequent calculations.

\section{Experimental results}

A series of measurements was taken to assess the accuracy of the experimental method and explore how an applied upwelling flow alters the thermal boundary layer structure. In section $4 \mathrm{a}$ we present measurements of the thermal boundary layer for free convection, before 


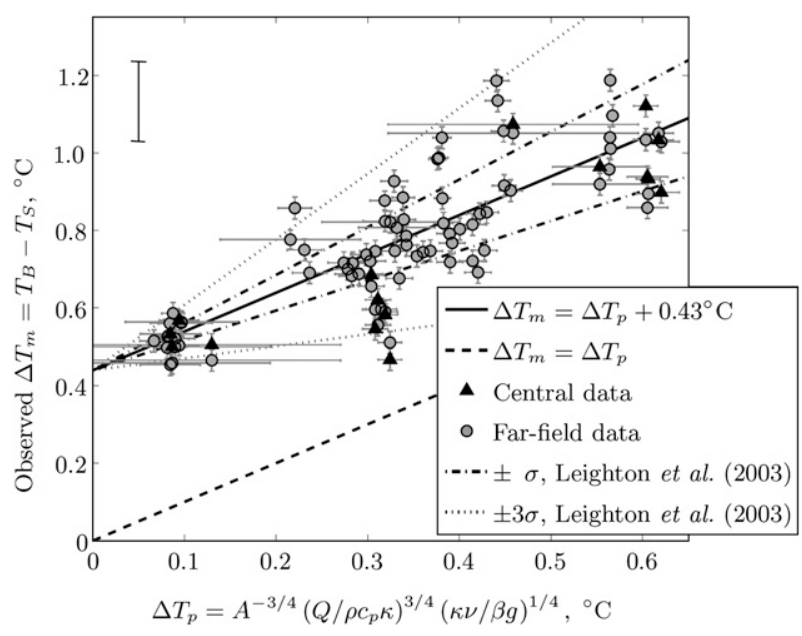

FIG. 7. Comparison of the observed bulk-skin temperature difference (measured using thermal camera and digital thermometer) with the theoretical prediction $T_{B}-T_{S}=A^{-3 / 4}\left(Q / \rho c_{p} \kappa\right)^{3 / 4}$ $(\kappa \nu / \beta g)^{1 / 4}$ given by (4), with $A=0.28$ obtained by a linear regression to the data. The black triangle symbols correspond to measurements in the center of the tank for free convection, with gray circles showing measurements taken in the far field at large distances from the upwelling jet. The dashed line shows the mean value expected from theory, with the solid line showing a linear relationship between measured values $\Delta T_{m}$ and predicted values $\Delta T_{p}$ obtained by fitting the constant $A$ and the intercept. Also shown are lines indicating the level of variability calculated by Leighton et al. (2003) due to turbulent flow variations, with the dashed-dotted and dotted lines indicating one and three standard deviations, respectively. Error estimates are decomposed into errors due to sensitivity of the estimates of $Q, T_{B}$, and $T_{S}$ (shown as one standard deviation error bars for each point), and a bound on the maximum possible bias between instruments (shown by the vertical bar in the top left corner of the plot).

moving on to consider the effects of an imposed upwelling flow in section $4 \mathrm{~b}$.

\section{a. Convective boundary layer with no imposed flow}

The experimental procedure was used to make measurements of a purely convective thermal boundary layer to provide a reference state, and also as a consistency check with previous studies (Katsaros et al. 1977; Leighton et al. 2003).

Figure 7 shows a comparison of the measured bulkskin temperature difference $T_{B}-T_{S}$ with the prediction (4) made by applying the four-thirds heat transfer law, with a fitted value of the constant $A$ (see below). The black triangle points correspond to measurements taken at the center of the tank for pure free convection (i.e., with no imposed upwelling flow). To maximize the number of samples under free convection conditions, we have also included measurements (gray circle symbols) from runs with an imposed upwelling flow but with the skin temperature measurements taken in the far field (dashed box in Fig. 6), approximately $8 \mathrm{~cm}$ from the upwelling jet at the center of the tank. The particle image velocimetry measurements show that vertical advection is weak in the far field, although there is a weak horizontal flow that may modify heat transfer. However, both sets of data appear to follow the same trend, suggesting that the far-field region is close to a free convective state and not strongly modified by the weak cross flow of the jet. A linear regression of the data yields the proportionality constant $A=0.28$, and intercept $T_{\text {offset }}=0.43^{\circ} \mathrm{C}$, with $95 \%$ confidence intervals $0.22<A<0.34$ and $0.38^{\circ} \mathrm{C}<T_{\text {offset }}<0.48^{\circ} \mathrm{C}$ obtained if the variation about the mean is assumed to be normally distributed. (Previous observations suggest a skewed distribution of skin temperatures for free convection, deviating slightly from a normal distribution, thus the quoted confidence intervals should be regarded as rough indications only, rather than formal statistical bounds). The value $A=0.28$ compares favorably with the value $A=0.29$ observed in the direct numerical simulations of Leighton et al. (2003), and is of similar order of magnitude, but it is slightly larger than the value $A=0.16$ observed by Katsaros et al. (1977). The better agreement of our measurements with the scaling of the direct numerical simulations is encouraging, as the simulations should approximate a "perfectly controlled" experiment. The explanation for the difference with Katsaros et al. (1977) is unclear, although Leighton et al. (2003) posed that the difference may be consistent with weak surface contamination in the Katsaros et al. (1977) experiments, which may result in a different fitted value of $A$. However, we expect that there is also surface contamination present in our experiments, as this is hard to avoid without an experimental methodology designed specifically for that purpose (e.g., McKenna and McGillis 2004).

The fitted intercept indicates that (4) consistently underestimates the observed bulk-skin temperature difference by approximately $0.43^{\circ} \mathrm{C}$. This offset is larger than the bounds on the measurement error of approximately $0.2^{\circ} \mathrm{C}$, suggesting that in addition to the possible measurement bias, there may also be some physical process present in our experiments that is not described by the theory. As a result, the experimental trend might not be suitable for extrapolation to values of $Q$ approaching zero. In this experiment, the Rayleigh numbers $\mathrm{Ra}_{h}=\beta g Q h^{4} / \rho c_{p} \kappa^{2} \nu$ based on the overall tank depth $h$ are relatively large, lying in the range $2 \times 10^{8}<\mathrm{Ra}_{h}<5 \times 10^{8}$. Convection cannot be maintained for very small Rayleigh numbers $\mathrm{Ra} \leqslant 10^{2}-10^{3}$, thus different physical processes will be important for small $Q$, which may change the trend. The discrepancy may be consistent with the effects of surface contamination 
observed in previous studies, and this and other possible factors are discussed in more detail in section 4c. The constant offset from the previously established theoretical scaling (4) motivates the use of an offset correction of $0.43^{\circ} \mathrm{C}$ in the later analysis, which is determined from the linear regression in Fig. 7.

The level of scatter of the data is also worthy of comment, and it may arise from a combination of sources, including measurement error and intrinsic physical variability due to the turbulent nature of the flow. Each skin temperature measurement is averaged over a 5-s period that is much shorter than the typical renewal time scale $\delta^{2} / \kappa \approx 1000 \mathrm{~s}$ of the skin layer and over an area smaller than a typical convection cell. Hence, each measurement effectively represents a sample of the turbulent flow, and intrinsic variability is expected about the mean state described in the theory. Leighton et al. (2003) observed physical variability in skin temperature with a standard deviation $\sigma=23 \%$ of the mean bulk-skin temperature difference, which is also consistent with variability measured by Katsaros et al. (1977). The dashed-dotted and dotted lines in Fig. 7 show $23 \%$ and $69 \%$ levels of variability about the mean trend based on the $\sigma$ and $3 \sigma$ levels, respectively, calculated by Leighton et al. (2003), after accounting for the $0.43^{\circ} \mathrm{C}$ offset. The scatter in the data is broadly consistent with this level of physical variability. (As a rough comparison, we expect $67 \%$ of samples of a normal distribution to lie within $\pm \sigma$ of the mean, and $99.7 \%$ within $\pm 3 \sigma$, although as discussed earlier we expect the distribution to deviate slightly from a normal distribution here.)

Figure 8 shows the raw output from a typical subsurface temperature profile. The persistent variation of $\pm 0.04^{\circ} \mathrm{C}$ corresponds to the precision of the instrument with the additional scatter of order $0.1^{\circ} \mathrm{C}$, believed to be due to electrical noise in the laboratory, contaminating the signal. An automated algorithm was used to estimate the vertical position of the air-water interface. Measurements in approximately the upper $0.3 \mathrm{~cm}$ have been taken in air (cross symbols) and are shown purely to illustrate the surface-detection algorithm. (The instrument does not measure temperature accurately in air.) The transition from readings taken in air to readings taken in water was characterized by a region of large vertical gradient in the temperature signal, as the probe pierced the interface. The position of the air-water interface was therefore estimated by taking the vertical coordinate of the tenth data point after the maximum value of $d T / d z$ was attained. The choice of 10 points is somewhat arbitrary, although it only makes a small difference to the estimate of the vertical position of the free surface, as each point corresponds to $0.001 \mathrm{~cm}$ of

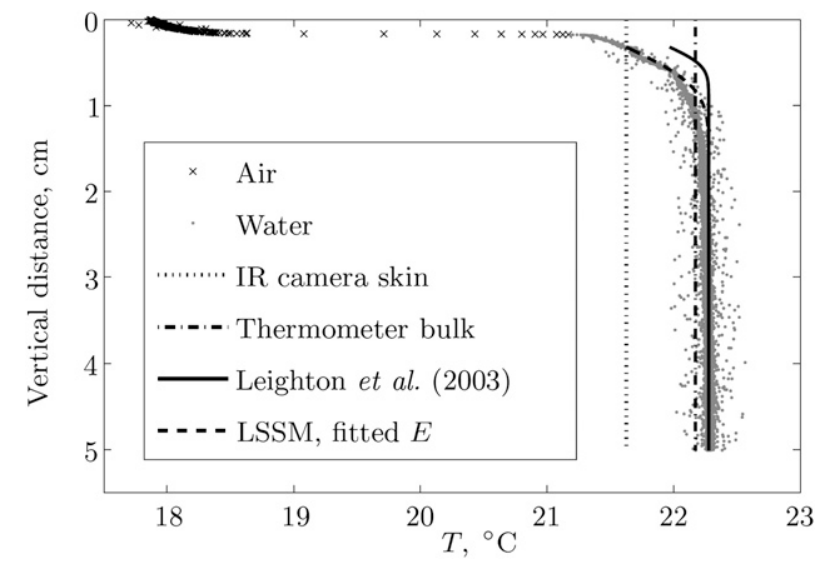

FIG. 8. Variation of subsurface temperature with depth. The measured temperature signal at each point is shown by symbols, with crosses denoting measurements in air and dots denoting measurements in water. The independent thermal camera measurement of the skin temperature is shown by a dotted line, and the thermometer measurement of the bulk temperature is shown by a dashed-dotted line. The solid curve shows the profile predicted by the Leighton et al. (2003) theory (14) estimating $E=\gamma$ by (16). The dashed curve corresponds to the theory (14) with $E$ fitted to match the observed bulk-skin temperature difference.

depth. This method does not give a good estimate of the skin temperature at the free surface: the difference between skin temperatures predicted by the algorithm and those measured by the thermal camera has an average of $-0.4^{\circ} \mathrm{C}$ with significant variability of order $0.3^{\circ} \mathrm{C}$ (some deviation is inevitable as a result of partial immersion of the probe as it pierces and deforms the interface). The temperature profile typically obtains the same value as the thermal camera estimate of skin temperature within a vertical distance of order $0.1 \mathrm{~cm}$ (which is, perhaps not coincidentally, comparable to the $0.05-\mathrm{cm}$ diameter of the glass-encased thermistor). The profile should, however, give an accurate measurement of the temperature variation with depth below the free surface. The data points from the remainder of the profile are inferred to be in water and are plotted using dot symbols. Visual inspection of the profiles suggests skin layer thicknesses of order $0.3-2.0 \mathrm{~cm}$, as expected for free convection, with thinner boundary layers observed for a larger surface heat flux. Attempts were also made to estimate skin layer thickness by applying an automated algorithm to examine differences between the temperature profiles and the bulk temperature, but the estimates proved overly sensitive to errors in the temperature measurements and so are not presented here. Also shown in Fig. 8 are comparisons to the temperature profiles predicted by a surface straining model. The temperature profile (14) predicted by Leighton et al. (2003) fares poorly, because the theory underestimates the magnitude of the 
Velocity vectors for upwelling jet near to surface

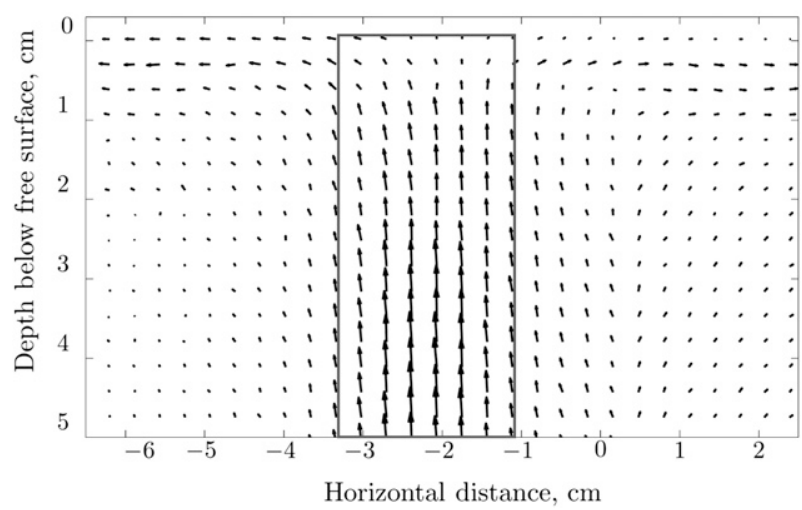

FIG. 9. Time-averaged velocity vectors calculated from PIV measurements for a laminar momentum jet with input volume flux $F=1.14 \mathrm{~cm}^{3} \mathrm{~s}^{-1}$. The vertical jet creates a local upwelling flow between $x=-3.2 \mathrm{~cm}$ and $x=-1.2 \mathrm{~cm}$, generating a diverging flow near to the surface. The mean vertical velocity is determined by taking a cross-sectional average at each height $z$ within the rectangular box.

measured bulk-skin temperature difference, consistent with the offset observed in Fig. 7. However, the comparison improves when the strain rate $E$ is fitted to match the observed bulk-skin temperature difference (dashed curve).

The measurements for free convection discussed in this section will help with the interpretation of the measurements with an additional imposed upwelling flow.

\section{b. Convective boundary layer with a divergence imposed near to the surface}

The vertical jet described in section 3 generated an upwelling flow of laminar character, although showing occasional intermittent bursts of unsteadiness. Figure 9 shows a plot of the time-averaged velocity vectors measured using PIV in a vertical section passing approximately through the center plane of the jet. The jet has a confined core flowing upward with weak entrainment of the exterior fluid, and it diverges radially as the free surface is approached. A 40-s average of the velocity seemed to yield a consistent estimate of the strain rate for repeated sets of measurements with each flow rate. However, small oscillations were occasionally observed in the jet trajectory and velocity, which leads to instantaneous measurements of the strain rate oscillating by $20 \%-50 \%$ around the mean value. Figure 10 shows a plot of the average vertical velocity $w(z)$ near the center of the jet, calculated using the methods described in section 3. Directly above the source $(z \sim-20 \mathrm{~cm})$, we observe an increase in vertical velocity-this is a consequence of the plane of measurement not coinciding precisely with the center of the jet. Closer to the surface,

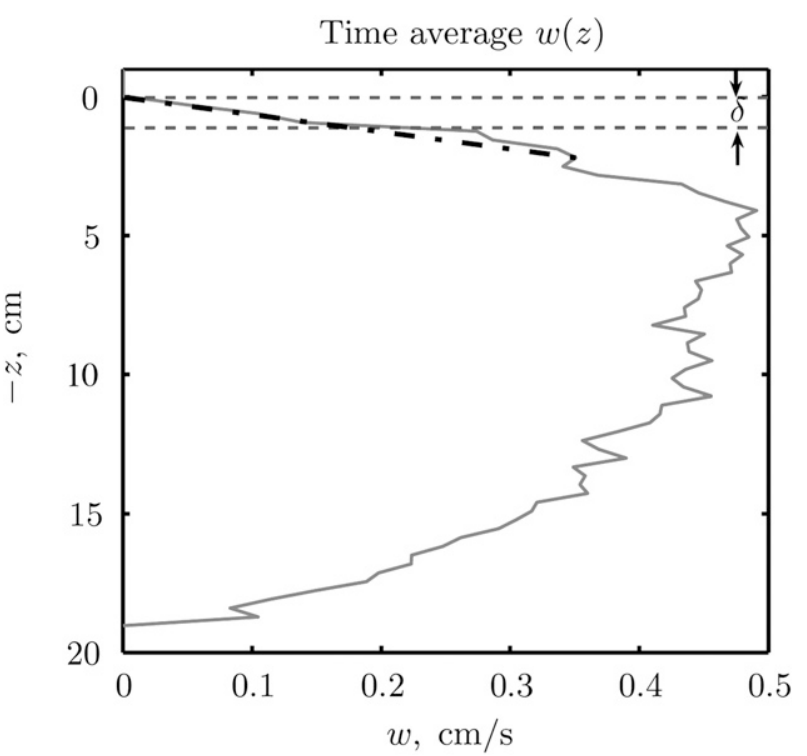

FIG. 10. Typical variation of mean vertical velocity $w$ with depth $-z$, for a jet with input volume flux $F=1.14 \mathrm{~cm}^{3} \mathrm{~s}^{-1}$. The velocity variation is linear close to the surface, so that the strain rate is approximately constant over the depth $\delta$ of the thermal boundary layer. A typical reference value of $\delta$ is marked by black dashed lines for comparison. The dashed-dotted line shows the linear velocity profile resulting from the strain rate estimated by (27).

we observe the expected vertical deceleration, with the vertical velocity tending to zero as we approach the free surface. The variation of vertical velocity $w$ is approximately linear in $z$ over the upper $2.5 \mathrm{~cm}$ of the profile. This length scale exceeds the typical thickness of the thermal boundary layer of order $0.3-2.0 \mathrm{~cm}$, so that we have an approximately constant strain rate within the thermal boundary layer. We estimate the strain rate by applying a linear finite difference across the upper $2 \mathrm{~cm}$ of the profile, so that

$$
\alpha=\frac{w(z=0 \mathrm{~cm})-w(z=-2 \mathrm{~cm})}{2 \mathrm{~cm}} .
$$

Different values of the mean strain rate were obtained by varying the jet momentum flux to give $\alpha=0.043$, $0.057,0.118,0.158$, and $0.432 \mathrm{~s}^{-1}$.

The imposed flow has a significant effect on the temperature observed both at and below the free surface. Figure 6 shows two examples of the instantaneous skin temperature measured with the infrared thermal camera. With no imposed flow (Fig. 6a), we observe signals consistent with convection cells, with upwelling creating regions of warm temperature bounded by narrow bands of colder temperature above downwelling plumes. These structures bear a striking resemblance to those observed in the direct numerical simulation of Leighton et al. (2003, see their Fig. 2). 
The addition of a vertical flow produces an obvious change in the skin temperature pattern, as seen in Fig. 6b. Upwelling of heat from the interior leads to a localized patch of warmer skin temperature above the center of the jet, as compared to the skin temperature in the far field. A patch of warm skin temperature is a persistent feature for all times when the upwelling jet is turned on. The convection cell structure appears to be suppressed within this warm patch, with no surface signature of downwelling plumes within the core of the jet. This is consistent with an imposed flow of large Péclet number $(\mathrm{Pe} \approx 4$ for the image shown) dominating the original free convection and stabilizing the thermal boundary layer.

To provide a quantitative comparison with the Osborne (1965) theory, the measured values of $\alpha$ and $Q$ were used to predict a value of the bulk-skin temperature difference for each experiment according to (17), with the skin layer thickness estimated by (5). The Osborne (1965) theory and the linear surface straining model give very similar predictions (Fig. 2), thus the corresponding comparison with the linear surface straining model (14) and (19) is broadly similar in nature. Figure 11 shows the bulk-skin temperature difference predicted by (17) compared to the value measured using the infrared camera and digital thermometer for each individual experiment. The results appear consistent with scatter about a linear trend, with the Osborne (1965) prediction (17) producing a consistent underestimate of the observed bulk-skin temperature difference by approximately $0.4^{\circ} \mathrm{C}$. This consistent underestimate is of very similar magnitude to the offset observed in Fig. 7 when comparing measurements of the bulk-skin temperature difference for free convection to a previously tested theory. The offset is also significantly larger than the order $0.2^{\circ} \mathrm{C}$ bound on absolute measurement accuracy, suggesting that in addition to any measurement bias there may be some other physical process at work not described by the theory. This will be discussed in more detail in section 4c. The black solid line in Fig. 11 shifts the theoretical prediction by an offset of magnitude $0.43^{\circ} \mathrm{C}$, determined by independently fitting the offset in the data for free convection from Fig. 7.

There is also significant scatter in the data about the mean trend. Part of the scatter might be explained by short-term variations of the strain rate from the temporal mean value of $\alpha$ applied in the theoretical prediction, with the plotted horizontal error bars being dominated by estimated variability in $\alpha$. It may also, however, reflect the variation of the environmental conditions in the laboratory, which would influence the instantaneous heat loss from the tank compared to the long-term mean. It is encouraging, however, that the

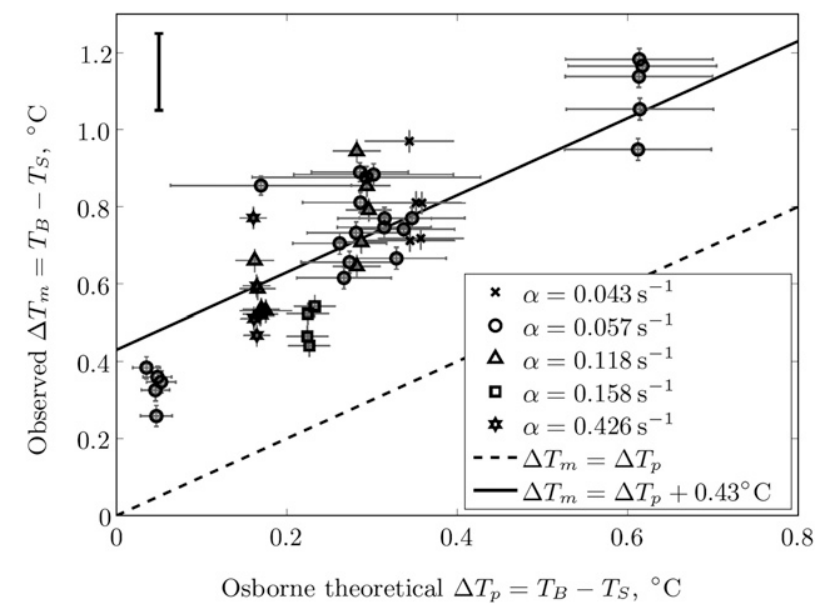

FIG. 11. Comparison of measured bulk-skin temperature difference $\Delta T_{m}$ to $\Delta T_{p}$ given by the theoretical prediction (17) of Osborne (1965), with $\delta_{e}$ calculated using (5). The observed skin temperature is measured using the thermal imaging camera, and the bulk temperature is measured by the digital thermometer. The dashed line $\Delta T_{m}=\Delta T_{p}$ and the solid line $\Delta T_{m}=\Delta T_{p}+0.43^{\circ} \mathrm{C}$ are also shown for reference, where the $0.43^{\circ} \mathrm{C}$ offset has been obtained by independently fitting the data for free convection in Fig. 7 to account for possible bias. (A corresponding fit to the data in this figure yields an offset of $0.42^{\circ} \mathrm{C}$ ). Error estimates for each point are decomposed into the possible persistent bias between instruments measuring bulk and skin temperatures (indicated by the black bar in the upper left corner), and individual measurement sensitivity indicated by error bars about each point. Error bars are calculated by a compound of the measurement sensitivity of $T_{S}$ and $T_{B}$, the confidence interval for $Q$ given by the linear regression, and the standard deviation of the observed level of variability of $\alpha$ about the mean state in the PIV measurements.

data appear to be scattered about the theoretical trend to within a constant offset.

The bulk-skin temperature difference illustrated in Fig. 11 depends on both the strain rate $\alpha$ and also the heat flux $Q$, which sets the strength of the free convection. The direct influence of strain on the skin layer is isolated by considering the variation of the nondimensional bulk-skin temperature difference $1 / \mathrm{Nu}$ with the Péclet number Pe, which characterizes the strength of the imposed flow compared to the initial free convective state. Note that the theoretical bulk-skin temperature difference for free convection yields a mean value $1 / \mathrm{Nu}=1$, so that the nondimensional formulation effectively scales out any dependence on $Q$ (individual measurements will, however, show scatter about this mean as a result of turbulent flow variations). To effectively capture variations of skin temperature compared to the free convective state measured in our experiments, we subtract the $0.43^{\circ} \mathrm{C}$ offset from all skin temperature measurements, so that the line of best fit to the free convection data in Fig. 7 reduces to $1 / \mathrm{Nu}=1$. Note 


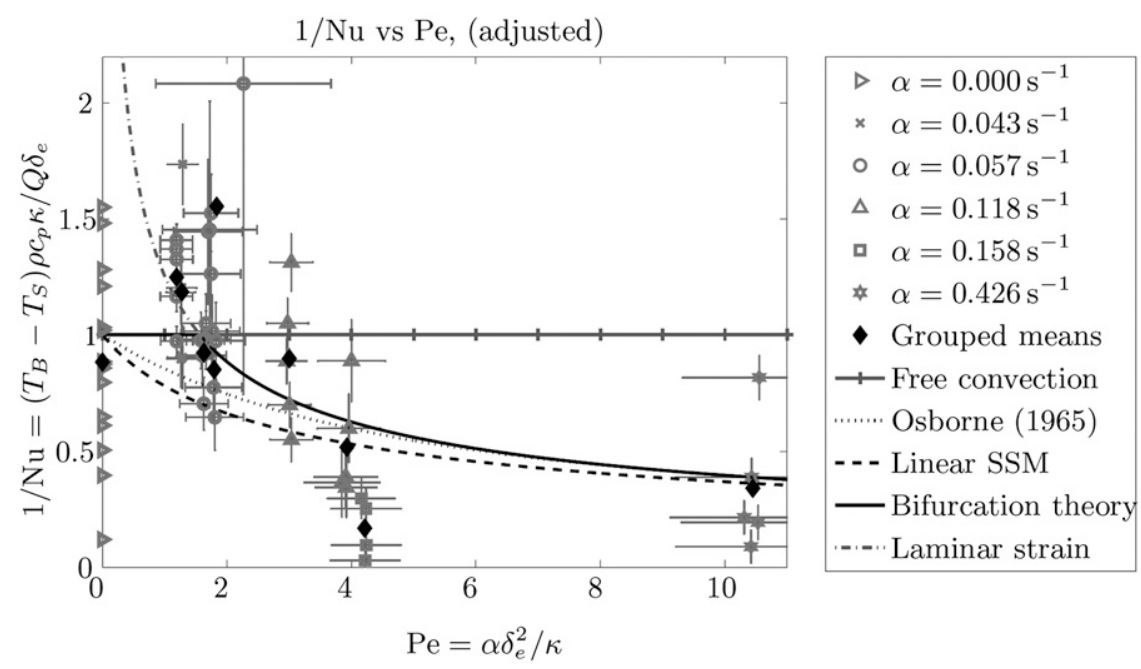

FIG. 12. Variation of inverse Nusselt number $1 / \mathrm{Nu}=\left(T_{B}-T_{S}\right) \rho c_{p} \kappa / Q \delta_{e}$ with Péclet number $\mathrm{Pe}=\alpha \delta_{e}^{2} / \kappa$, using skin temperatures adjusted by a $0.43^{\circ} \mathrm{C}$ offset to remove bias (see text for details). All values of $\delta_{e}$ are estimated using (5). The black diamond points represent mean values for groups of data from consecutive experiments repeated at similar conditions (each group contains four or five points with the same value of $\alpha$, and values of $T_{B}$ lie within a range of $0.4^{\circ} \mathrm{C}$ ). Also shown are curves corresponding to the predictions of the Osborne (1965) theory. The bifurcation theory based on instability coincides with the free convection theory for $\mathrm{Pe}<\pi / 2$, and the laminar straining flow result for $\mathrm{Pe}>\pi / 2$. The theories accounting for strain appear to give a better description than the theory describing free convection alone. Five data points for $\alpha=0.057 \mathrm{~s}^{-1}$ are missing in this plot, because the offset correction generates a negative value of $\mathrm{Nu}$. These missing data points correspond to data at small heat fluxes, so that the measurement accuracy bounds allow for substantial variation of $1 / \mathrm{Nu}$.

that this correction will eliminate any measurement bias between the instruments, but it may also remove some physical contributions to the bulk-skin temperature difference. Figure 12 shows the variation of $1 / \mathrm{Nu}$ with $\mathrm{Pe}$, using the adjusted values of skin temperature. Also shown are curves corresponding to the predictions of the Osborne (1965) theory (T5a, dotted curve); the linear surface straining model (T5b, black dashed curve); the bifurcation theory (21) and (22), and the black solid curve; the prediction (4) for free convection, which ignores the influence of the straining flow (gray solid curve with cross symbols); and the prediction (14) for a pure straining flow with $E=\alpha$, ignoring free convection (gray dashed-dotted curve). The data show considerable scatter, in part because of the time-dependent variation of convection about the mean state and the time-dependent variability of $\alpha$ about the mean value. However, for large Péclet numbers, the trend of variation of skin temperature is generally better described by theories incorporating strain (black dotted, dashed, and solid curves) than by the free convection theory alone (shown by a gray line with cross symbols). This is consistent with the straining flow significantly influencing the skin temperature at large Péclet numbers, within the considerable scatter in the data. For $\mathrm{Pe}=O(1)$, the free convection and straining theories yield similar predictions, and it is hard to distinguish between them.

To examine this point more carefully, a residual analysis was carried out for each theory by examining the differences between each data point and the corresponding model to check for additional trends in the residuals not explained by the model. Figure 13 shows a plot of each set of residuals as a function of Péclet number for theories describing pure free convection (Fig. 13a), the Osborne (1965) theory (Fig. 13b), the linear surface straining model (Fig. 13c), and the bifurcation theory based on instability (Fig. 13d). In an attempt to quantify whether trends in the residuals are statistically significant, a linear regression was applied to the residuals against Pe to determine the likelihood that the residuals could be explained by a normal distribution with no trend. The assumption of normally distributed residuals and a comparison to a linear trend was applied in absence of detailed knowledge of the expected distribution of residuals, and so the probability values should only be interpreted as rough indicators of trends rather than part of a formal statistical test. The resulting statistics are summarized in Table 2. The extreme low $p$ value for the free convection theory indicates evidence of a trend in the residuals, as is apparent in Fig. 13a. The theories 
(a)

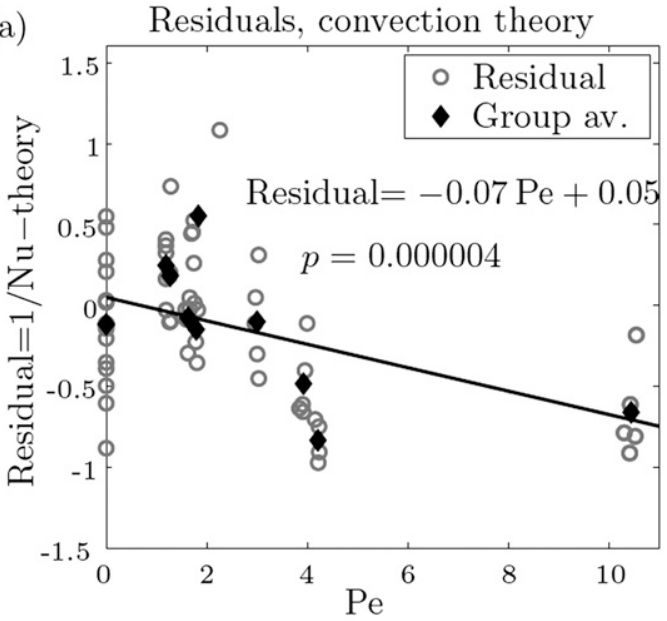

(c)

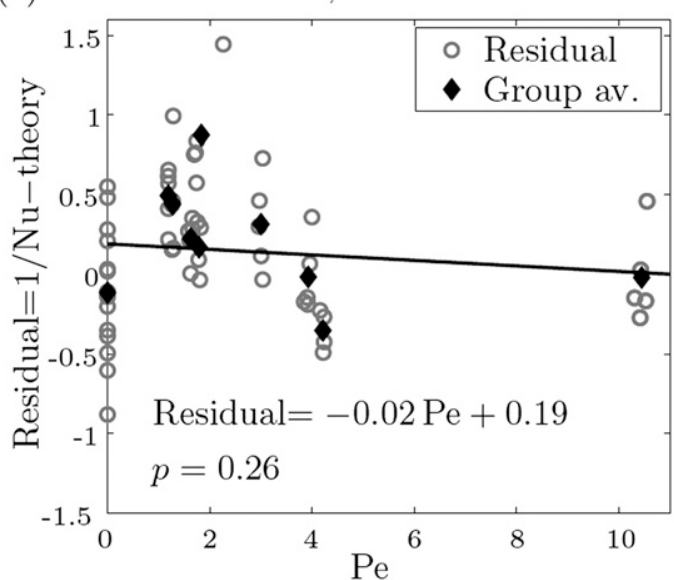

(b) Residuals, Osborne theory

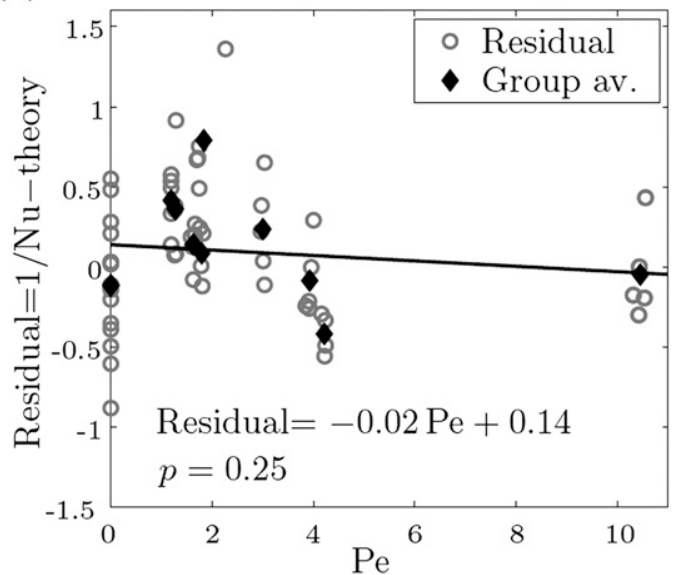

(d) Residuals, bifurcation theory

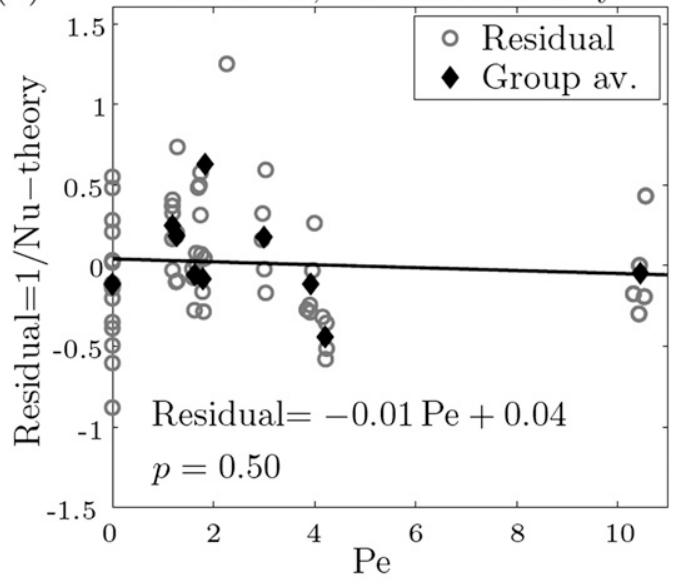

FIG. 13. Variation with Pe of the residual between data and model for (a) pure free convection using (4), (b) the Osborne (1965) theory (T5a), (c) the linear surface straining model (T5b) and (d) the bifurcation theory based on instability and described by (21) and (22). Gray circles show individual data points, with the black solid diamonds showing the grouped means described in Fig. 12. Solid lines show the results of a linear regression of residuals against Pe. The resulting trend lines are stated in each figure, along with the probability of no significant trend in the residuals according to an $F$ test (see main text).

incorporating strain all yield moderate $p$ values (if the data were normally distributed, these $p$ values would correspond to no statistically significant trend in the residuals at an $80 \%$ confidence level), suggesting that these theories give a better description of the trend in the data than the free convection theory alone. The strongest fit to the data (highest $p$ value, lowest $R^{2}$ ) is provided by the bifurcation theory (21) and (22) based on instability, with the Osborne (1965) and linear surface straining models (Figs. 13b and 13c) both showing evidence of weaker agreement for small Péclet numbers.

TABLE 2. Summary of statistics for linear regressions of the residuals of data compared to theory (Res) against Pe. A $95 \%$ confidence interval is indicated for both slope and intercept of each trend line, along with an $R^{2}$ value for fit to the linear trend and a $p$ value estimating the probability that the residuals are explained by no trend in the underlying distribution (using an $F$ test). Note that the confidence intervals and $p$ values require an assumption of normally distributed error and thus should be treated as rough indicators only.

\begin{tabular}{llcc}
\hline \hline \multicolumn{1}{c}{ Theory } & \multicolumn{1}{c}{ Linear regression } & $p$ value & $R^{2}$ value \\
\hline Convection & Res $=(-0.07 \pm 0.03) \mathrm{Pe}+0.05 \pm 0.13$ & $4 \times 10^{-6}$ & 0.29 \\
Osborne & $\operatorname{Res}=(-0.02 \pm 0.03) \mathrm{Pe}+0.14 \pm 0.13$ & 0.25 & 0.02 \\
LSSM & $\operatorname{Res}=(-0.02 \pm 0.03) \mathrm{Pe}+0.19 \pm 0.13$ & 0.26 & 0.02 \\
Bifurcation theory & $\operatorname{Res}=(-0.01 \pm 0.03) \mathrm{Pe}+0.04 \pm 0.12$ & 0.50 & 0.01 \\
\hline
\end{tabular}




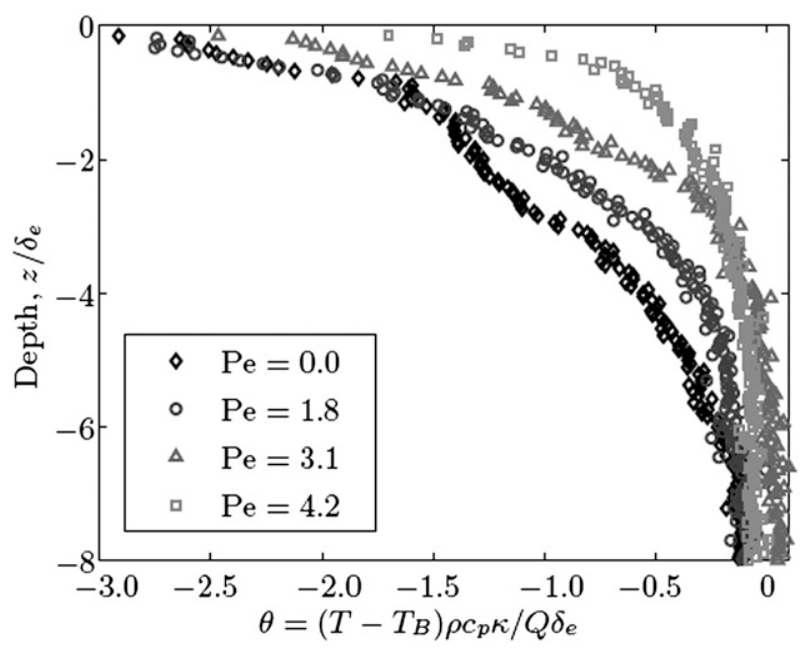

FIG. 14. Variation of the nondimensional subsurface temperature, $\theta=\left(T-T_{B}\right) \rho c_{p} \kappa / Q \delta_{e}$, with scaled depth $z / \delta_{e}$ for a range of Péclet numbers, where $\delta$ is given by (5) to scale out the dependence on the initial free convection. Each temperature profile has been smoothed with a $0.01-\mathrm{cm}$ (10 point) box filter to remove noise, with every tenth point plotted over the upper section of the profile. As Pe and hence the vertical advection increases, the thermal boundary layer becomes thinner and the bulk-skin temperature difference is reduced.

The variation in skin temperature is accompanied by a corresponding change to the subsurface thermal boundary layer. Figure 14 shows the measured temperature variation with depth for four different applied strain rates. The nondimensional formulation has been used to remove the influence of $Q$ and isolate the influence of strain. The profiles are consistent with a reduction in the bulk-skin temperature difference as the Péclet number increases. The profiles also suggest an apparent reduction in the measured boundary layer thickness as Pe increases, with the temperature variation confined to a narrower region close to the surface. This is qualitatively consistent with the asymptotic structure for large Péclet numbers considered in section $2 \mathrm{~d}$ and appendix B. For smaller Péclet numbers, the profiles show some evidence of a complex boundary layer structure, with a kink in several of the profiles near to $z / \delta_{e}=-1$. It is interesting that this kink occurs close to the depth of the effective diffusive layer for a free convective boundary layer, so that the kink could perhaps be associated with convective instability of the boundary layer. However, the full underlying physical explanation is not clear at this stage.

\section{c. Discussion of possible causes of the observed temperature offset}

Figures 7, 11, and 12 show that the theoretical solutions underpredict the bulk-skin temperature differ- ences observed in the experiments by approximately $0.4^{\circ} \mathrm{C}$, both with and without the imposed flow of the jet. A consistent offset for all experiments suggests the possibility of measurement bias, with different instruments used to measure the bulk and skin temperatures. However, the magnitude of the $0.4^{\circ} \mathrm{C}$ underestimate is significantly larger than the estimated experimental measurement accuracy based on the quoted manufacturer's standard, which compounds to approximately $0.2^{\circ} \mathrm{C}$ in the worst case, and the stirring test comparison suggests biases are only of order $0.03^{\circ} \mathrm{C}$. We are unable to firmly rule out the possibility of drift in the accuracy of the bulk and skin temperature measurements during the course of the experiments. However, the independent temperature profile measurements also suggest that skin temperatures are between $0.5^{\circ}$ and $1.1^{\circ} \mathrm{C}$ colder than predicted by the theories (although this estimate is less reliable, because of the interface deformation effects discussed in section $4 \mathrm{a}$ ).

A $0.4^{\circ} \mathrm{C}$ offset between measurement and theory could arise from errors in the heat flux $Q$ applied in the theory, with (4) suggesting an underestimate of $Q$ by approximately $130 \mathrm{~W} \mathrm{~m}^{-2}$ would be required to explain the discrepancy. There are several possible sources of error in $Q$. The integral heat budget has neglected heat loss through the sides of the tank, but this leads to an overestimate of $Q$ because the water temperatures were warmer than the ambient air temperature; therefore, this error is of the wrong sign to explain the discrepancy. It is possible that the water may be heated slightly during a cycle through the pump, but this effect cannot explain the discrepancy during free convection cases where no water is cycled through the pump. An alternative unaccounted source of heating of the water may be from absorbed radiation incident from the artificial lighting in the laboratory. However, a conservative estimate yields radiative fluxes from the lamps of less than $15 \mathrm{~W} \mathrm{~m}^{-2}$ based on bulb spacing and radiative power output, and we expect the net energy flux absorbed by the water to be smaller than this amount.

As an additional comparison, the temperature profiles $T(z)$ were examined to check whether our estimated values of $Q$ are of the right order of magnitude. The temperature gradient near to the free surface was estimated by applying a linear regression to each set of temperature profile measurements over the depth range $-0.13 \mathrm{~cm}<z<-0.03 \mathrm{~cm}$ below the free surface position estimated by our automated algorithm. The linear regression then yields a second independent estimate of the heat flux from $Q_{\text {reg }}=-\rho c_{p} \kappa \partial T / \partial z$. The range of $z$ values were chosen to yield an approximately constant temperature gradient over the interval, with a visual inspection showing negligible curvature for all of the 
temperature profiles (applying the regression at larger depths leads to smaller values of $Q_{\text {reg }}$ because of the variation of the temperature profile with depth). Comparing values of $Q_{\text {reg }}$ to the values $Q$ estimated from bulk temperature, we obtain a line of best fit $Q_{\text {reg }}=$ $1.2 Q+39 \mathrm{~W} \mathrm{~m}^{-2}$ after omitting five spurious estimates with unrealistically large values $Q_{\text {reg }}$ of order 400 $600 \mathrm{~W} \mathrm{~m}^{-2}$ (visual inspection suggests these large values are likely because of the inaccuracy of the free surface detection algorithm). Despite the inherent difficulties in making the temperature profile measurements very close to the free surface (discussed in section 4a), which make the estimates of $\partial T / \partial z$ less reliable, it is encouraging that the two estimates are of similar magnitude, with the intercept of the fit suggesting an average offset of $40 \mathrm{~W} \mathrm{~m}^{-2}$ between the two methods. This is equivalent to a temperature offset of $0.17^{\circ} \mathrm{C}$ using (4). These independent estimates provide further indication that the observed discrepancy between measurements and theory cannot be explained entirely by measurement errors in $Q$.

The observed underestimate of the bulk-skin temperature difference might alternatively arise because of some aspect of the physics in the laboratory experiment that is not described or modeled in the theory. One possibility is that the water flow causes a modification of the water-to-air heat transfer over the upwelling jet, as a result of a local modification of the skin temperature and the airflow pattern above the surface. However, the local change in heat flux required to explain the offset ranges between increases of $60 \%$ and $900 \%$ of the value of $Q$ inferred from the bulk temperature in the tank. It would be unlikely that such a large percentage change in $Q$ could arise as a result of a change of less than $20 \%$ in the temperature difference between the air-water interface and the far-field atmospheric temperature. In addition, this effect does not explain the consistent underprediction of bulk-skin temperature difference for the free convective case. Another process not described by the theory is Marangoni convection (Pearson 1958), which arises from an instability due to the variation of the surface tension of the fluid with temperature. However, this effect would contribute to a discrepancy of the opposite sign to the one observed.

We believe the most likely explanation for observing a colder skin temperature than expected is the influence of surface contamination. The tap water used in experiments may contain nonnegligible quantities of surfactant, surface contaminants may be deposited on the surface from the atmosphere during the course of the experiments, and the PIV seeding material also introduces artificial contamination. Such surface contamination can significantly affect transfer processes across an air-water interface (e.g., McKenna and McGillis
2004), with previous laboratory measurements (Saylor et al. 2000) and direct numerical simulations (Handler et al. 2003) showing that the skin temperature can be reduced by as much as $1^{\circ} \mathrm{C}$. These effects are not included directly in the theoretical predictions (4), (17), and (14), and so the discrepancy with the measured skin temperature might be consistent with the previous observations of Saylor et al. (2000) and Handler et al. (2003). In addition, comparing the skin temperature field for free convection (Fig. 6a) to similar measurements for clean and artificially contaminated interfaces in Fig. 2 of Saylor et al. (2000), we see that our measurements qualitatively show better resemblance with the long wavelength variability observed for a contaminated interface than the shorter wavelength variability for a clean interface. However, it is unclear whether surface contamination would lead to a constant offset, or some complicated variation that depends on the flow conditions. It is also possible that the overflowing of the inner tank may advect some surface contamination away during runs with an upwelling jet, although it is unclear whether horizontal velocities of order $0.1 \mathrm{~cm} \mathrm{~s}^{-1}$ and smaller were sufficient to remove all the surface contamination. In addition, the overflow would not remove water soluble surfactants, such as those contained in tap water and in the particle solution used for the particle image velocimetry measurements.

The additional processes described above and others not considered here may be present, modifying the overall magnitude of the cool skin effect leading to an apparent offset. However, it is encouraging that, for upwelling flows that are strong compared to the initial free convective state, the changes in skin temperature with strain appear consistent with the variation in skin temperature predicted by the theoretical models. This emphasizes that the processes of advection and diffusion described in the theory control the transfer of heat across the molecular thermal boundary layer.

\section{Discussion}

Three theories have been considered that describe the influence of a straining flow acting directly within the skin layer. Osborne (1965) calculated the change in skin temperature due to a laminar straining flow added within an effective diffusive layer of fixed thickness $\delta_{e}$, where $\delta_{e}$ is determined by the initial turbulent state. We have also posed a linear extension of a surface straining model that calculates temperature changes due to the sum of strains generated by the initial turbulence and the extra imposed upwelling flow. Comparison of the Osborne (1965) theory and the linear surface straining model shows only a small difference in the predicted skin 
temperature, despite the different assumptions employed by the two theories. Finally, for cases with free convective turbulence, we proposed a theory based on the idea of instability generating a bifurcation in the flow state with strong upwelling flows acting to create a narrow thermal boundary layer that suppresses buoyancy-driven instability, but weaker upwelling flow allowing instability and subsequent free convection. It may also be possible to extend the principle of the bifurcation theory to describe shear-driven turbulence generated by winds, with strong upwelling resisting the development of shear instability by enhancing vorticity confinement within the viscous boundary layer. However, the details of such an extension have not been considered here.

Each of the theories yield distinct physical limits that occur for different limiting values of the dimensionless Péclet number $\mathrm{Pe}=\alpha \delta_{e}^{2} / \kappa$. The Péclet number measures the ratio of advection by the imposed flow relative to diffusion across the skin layer of thickness $\delta_{e}$ that would arise in the presence of turbulence alone. In our laboratory experiments, $\delta_{e}$ was controlled by free convection; however, a different choice of $\delta_{e}$ for the Osborne model or $\gamma$ in the linear surface straining model allows for the description of other sources of turbulence, such as shear-driven flows generated by winds.

For large Péclet numbers, the advection is strong compared to the initial free convection, and all theories approach an asymptotic limit where the skin temperature is independent of the strength of the initial free convection (i.e., independent of $\delta_{e}$ for Osborne's model and independent of the background strain rate $\gamma$ in the linear surface straining model). The theories also predict a corresponding thinning of the thermal boundary layer for strong upwelling flow. Heat transport from the interior is dominated by advection, and so the skin temperature is controlled by a steady-state balance between vertical advection of heat into the boundary layer and loss of heat to the atmosphere. The thermal boundary layer then adjusts its thickness to supply the necessary conducted heat flux at the air-water interface, giving a theoretical asymptotic scaling $\sqrt{\kappa / \alpha}$ for the reduced boundary layer thickness, consistent with the scaling for a laminar straining flow with no convection. The thermal boundary layer effectively adjusts to maintain an $O(1)$ Péclet number based on the reduced boundary layer thickness; that is, for a strong straining flow, all three theories predict a balance between advection and molecular diffusion. The measured temperature profiles give some qualitative support for a reduction in boundary layer thickness.

The laboratory measurements also demonstrate that these strong upwelling flows do indeed generate an increase in skin temperature of a cooling body of water.
For large Péclet numbers, the mean trend for variation of the skin temperature with Péclet number appears to be better described by the theories incorporating strain than by the free convection scaling alone. However, there is considerable scatter in the data about the mean trend, likely due to a combination of physical flow variations, variation of environmental conditions about the mean state, and measurement noise. In addition, the theories also consistently underpredict the observed bulk-skin temperature difference by about $0.4^{\circ} \mathrm{C}$, for both free convection and with an imposed flow. A variety of factors were considered in an attempt to explain this offset, which may be due to some element of physics not described by the theory. In particular, the magnitude of the underprediction is comparable to the previously observed influence of surface contamination (Saylor et al. 2000; Handler et al. 2003), which was likely also present in our experiments. However, we were unable to confirm whether this is the cause of the offset.

For small Péclet numbers, the imposed advection is relatively weak compared to the initial free convection, and the theories predict a different regime of behavior. Theoretical calculations for time-dependent flows of long period $\left(\omega \delta^{2} / \kappa \ll 1\right)$ show that a previous result by Osborne (1965) for sinusoidally varying flows can be extended to flows of general time dependence. A corresponding result has also been derived for the linear surface straining model. Hence, both models suggest that small Péclet number flows of long period produce only a small percentage change in the bulk-skin temperature difference by direct straining in the skin layer. The variation occurs in a quasi-steady fashion at leading order. Both models assume that the upwelling flow can simply be added to the initial free convection without significantly modifying the free convective flow, an assumption that still requires further experimental evaluation for flows that have small Péclet numbers or are unsteady. The bifurcation theory based on instability predicts no change in the skin temperature for small Péclet number flows. The experimental measurements of skin temperature for small and moderate Péclet numbers $(\mathrm{Pe} \lesssim 2$ ) were unable to firmly distinguish between each of these theories, perhaps because the predicted signals are small compared to our experimental accuracy. However, it is interesting to note that the residual analysis indicates the best overall fit to the data is provided by the bifurcation theory based on instability. This suggests an avenue for future investigation, both experimentally and theoretically, to confirm whether the skin temperature changes gradually as the flow strength increases, or whether the change is initiated by a bifurcation at a critical value of the Péclet number. Any experimental study of this would benefit 
from efforts to control surfactants and longer sampling or averaging times.

The theoretically predicted behavior has possible implications for oceanic flows. If we assume that the Osborne (1965) and linear surface straining models yield an accurate description of small Péclet number flows, then (T7) implies that the fractional change in bulk-skin temperature difference is bounded at $O(\mathrm{Pe})$ for steady flows or slowly varying flows with $\mathrm{Pe} \ll 1$ and $\omega \delta^{2} / \kappa \ll 1$. A relatively large Péclet number is therefore required for an upwelling flow to generate an appreciable change of the skin temperature by direct straining of the skin layer. (Note that this would also be the case for the theory based on instability, which predicts no change from free convection scalings for small Pe.) This is illustrated by the following idealized example, motivated by the influence of the large-scale flow generated by internal gravity waves. Measurements of large amplitude internal waves have observed strains of amplitude $\alpha_{0}=0.002 \mathrm{~s}^{-1}$ in the surface mixed layer (Gasparovic et al. 1988). If the undisturbed skin layer thickness is $\delta \approx 0.1 \mathrm{~cm}$ for turbulence driven by wind shear and $\kappa \approx 10^{-3} \mathrm{~cm}^{2} \mathrm{~s}^{-1}$, then we obtain a Péclet number $\mathrm{Pe}=0.02$. An internal wave has a typical frequency of $\omega \leq 10^{-2} \mathrm{~s}^{-1}$, yielding $\mathrm{StPe} \leq 0.1$, so that the period is long compared to the thermal diffusion time, and the Osborne (1965) and linear surface straining models can be applied in a quasi-steady fashion. If we assume that the strain varies smoothly in time between $\pm \alpha_{0}$ (the exact form of time-dependence is unimportant), then application of the Osborne (1965) result (T7a) with an ocean-to-atmosphere heat flux of $Q=100 \mathrm{~W} \mathrm{~m}^{-2}$ leads to a negligibly small variation of only $0.249^{\circ} \mathrm{C} \leq T_{B}-T_{S}(t) \leq 0.251^{\circ} \mathrm{C}$ in the bulk-skin temperature difference compared to the value $T_{B}-T_{S}=$ $0.25^{\circ} \mathrm{C}$ obtained with no imposed flow $(\alpha=0)$. The linear surface straining model gives $0.248^{\circ} \mathrm{C} \leq T_{B}-T_{S}(t) \leq$ $0.252^{\circ} \mathrm{C}$, which is similarly small. We therefore expect the large-scale flow due to internal waves to generate negligible change in skin temperature by direct straining of the skin layer, unless the three theories are all inaccurate for unsteady small Péclet number flows. In particular, this signal of order $\pm 0.002^{\circ} \mathrm{C}$ predicted for direct straining of the skin layer is significantly smaller than the order $\pm 0.05^{\circ} \mathrm{C}$ variability observed in the skin temperature accompanying internal waves in the absence of diurnal warming (Zappa and Jessup 2005; Farrar et al. 2007). This leads to the hypothesis that some other process must contribute to the observed signal, such as the modulation of near-surface turbulence, surface contamination levels, or surface waves, or else some process that leads to an amplification of the strain experienced in the skin layer. For example, the regions of converging flow generated by internal waves can lead to a shortening of the wavelength of surface waves, which may indirectly lead to modification of the skin temperature.

Skin layer straining may, however, be important for large Péclet number flows. For example, Peirson and Banner (2003) and Turney et al. (2005) measured surface divergences of approximately $1-10 \mathrm{~s}^{-1}$ for microbreaking surface waves, yielding $\mathrm{Pe} \sim 10-100$ for a $0.1-\mathrm{cm}$ skin layer. In addition, flow separation can produce high vorticity wavelets with $\mathrm{Pe} \sim 200$ (Csanady 1990), and white-capping can also generate significant strains. On the basis of the experimental data, we expect the variation of the skin temperature for steady large Péclet number flows to be reasonably well described by (14), which is the asymptotic limit of each of the posed theories for $\mathrm{Pe} \gg 1$, up to the influence of surface contamination.

The results can also be modified to infer consequences for the transfer of highly soluble gases subject to a condition of constant flux between the ocean and atmosphere. If $\delta$ is the thermal boundary layer thickness, determined by the level of subsurface turbulence, then the gas boundary layer thickness scales as

$$
\eta \propto \mathrm{Le}^{-n} \delta=\left(\frac{D}{\kappa}\right)^{n} \eta
$$

where the Lewis number is Le $=\kappa / D, D$ is the gas diffusivity, and the exponent $1 / 2 \leq n \leq 2 / 3$ varies depending on the degree of surface contamination (Jähne et al. 1979). The nondimensional representation presented in section $2 \mathrm{~d}$ can then be applied by replacing temperature by gas concentration, defining a corresponding gas flux to replace the heat flux $Q$, and using a Péclet number based on gas diffusivity $\mathrm{Pe}_{D}=\alpha \eta^{2} / D$. The ratio of gas Péclet number to thermal Péclet number then depends critically on the ratio of the gaseous and thermal boundary layer thicknesses. If the interface has no surface contamination, then we expect a free-slip boundary condition, so that the exponent $n=1 / 2$. From (28) we see that the resulting thermal and gas Péclet numbers are of the same order of magnitude, so that heat and gas transfer behave in the same way. For contaminated interfaces we have $n>1 / 2$ and, if $\mathrm{Le}=\kappa / D \gg 1$, then the gas Péclet number is significantly smaller than the thermal Péclet number, and hence straining has a weaker effect on surface gas concentration than it does on temperature.

Acknowledgments. We thank Grae Worster, Colm Caulfield, Gregory Lane-Serff, and two anonymous reviewers for helpful comments on earlier versions of this work, and Grae Worster for the suggestion to consider the instability-based theory. The work was initiated during the Woods Hole Oceanographic Institution Geophysical 
Fluid Dynamics Program, where AJW was supported by a fellowship of the program. CC was supported by NSF Grant OCE-82633900. JTF was supported in part by The Penzance Endowed Fund in Support of Assistant Scientists. CJZ was supported by NSF Grant OCE-0425395 and the Office of Naval Research Young Investigator Program Grant N00014-04-1-0621. Additional funding for this research came from the U.S. Office of Naval Research through the Coupled Boundary Layer Air-Sea Transfer Departmental Research Initiative (Grants N00014-05-10090 and N00014-05-1-0036).

\section{APPENDIX A}

\section{Time-Dependent Solutions for Small Péclet Numbers}

For small Péclet numbers, we can apply a perturbation expansion in $\mathrm{Pe} \ll 1$ to obtain leading order solutions of the unsteady nondimensional heat equation (T1) for both the Osborne (1965) and linear surface straining models. We assume time-dependent forcing $\alpha=\alpha_{0} f(\omega t)$ with $|f(\hat{t})| \leq 1$, and the Péclet number is defined by $\mathrm{Pe}=\alpha_{0} \delta_{e}^{2} / \kappa$. We seek solutions via a regular perturbation expansion, valid for relatively weak advection $\left(\mathrm{Pe}=\alpha_{0} \delta_{e}^{2} / \kappa \ll 1\right)$ and for motions of long period compared to the diffusion time so that $\mathrm{St} \mathrm{Pe}=\omega \delta_{e}^{2} / \kappa \ll 1$ with $\mathrm{St}=\omega / \alpha=O(1) .^{2} \mathrm{We}$ consider solutions for both the Osborne (1965) and linear surface straining models in turn, and we find that both models yield a bulkskin temperature difference that varies in a quasi-steady fashion at leading order.

\section{a. Osborne model}

For the Osborne (1965) model, the nondimensional heat equation (T1) and (T2a) give

$$
\operatorname{Pe}\left[\operatorname{St} \frac{\partial \theta}{\partial \hat{t}}-f(\hat{t}) \hat{z} \frac{\partial \theta}{\partial \hat{z}}\right]=\frac{\partial^{2} \theta}{\partial \hat{z}^{2}},
$$

which is to be solved in conjunction with the boundary conditions (T3a) and (T4a). We seek solutions of the form $\theta=\theta_{0}(\hat{z}, \hat{t})+\operatorname{Pe} \theta_{1}(\hat{z}, \hat{t})+\operatorname{Pe}^{2} \theta_{2}(\hat{z}, \hat{t})+\cdots$ and solve at each order in Pe. The boundary conditions yield

$$
\begin{aligned}
& \theta_{0}=\theta_{i}=0 \quad \text { at } \quad \hat{z}=-1, \quad \text { and } \\
& \frac{\partial \theta_{0}}{\partial \hat{z}}=-1, \\
& \frac{\partial \theta_{i}}{\partial \hat{z}}=0 \quad \text { at } \quad \hat{z}=0, \quad i=1,2, \ldots
\end{aligned}
$$

\footnotetext{
${ }^{2}$ The limit St $\mathrm{Pe} \rightarrow 0$ is a singular perturbation in time, but we look for long time solutions and thus ignore the initial transient spin-up effects this singular perturbation would give.
}

At leading order (A1) gives

$$
\frac{\partial^{2} \theta_{0}}{\partial \hat{z}^{2}}=0
$$

with solution

$$
\theta_{0}=-1-\hat{z}
$$

which represents the temperature variation from conduction only across the effective diffusive layer.

The effects of advection enter at $O(\mathrm{Pe})$, where, after substituting for $\partial \theta_{0} / \partial \hat{z}$, we obtain

$$
\frac{\partial^{2} \theta_{1}}{\partial \hat{z}^{2}}=f(\hat{t}) \hat{z}
$$

which has solution

$$
\theta_{1}=f(\hat{t})\left(\frac{1+\hat{z}^{3}}{6}\right)
$$

so that the leading order effects of time-dependent advection occur in a quasi-steady fashion.

The first influence of the unsteady term $\partial \theta / \partial \hat{t}$ is at $O\left(\mathrm{StPe}^{2}\right)$. Eliminating $\theta_{1}$ from (A1) yields

$$
\frac{\partial^{2} \theta_{2}}{\partial \hat{z}^{2}}=\operatorname{St} \frac{d f}{d \hat{t}}\left(\frac{1+\hat{z}^{3}}{6}\right)-[f(\hat{t})]^{2} \frac{\hat{z}^{3}}{2},
$$

with solution

$$
\theta_{2}=\operatorname{St} \frac{d f}{d \hat{t}}\left(\frac{\hat{z}^{5}+10 \hat{z}^{2}-9}{120}\right)-[f(\hat{t})]^{2} \frac{1+\hat{z}^{5}}{40} .
$$

The nondimensional bulk-skin temperature difference is given by $1 / \mathrm{Nu}=-\theta(0, \hat{t})$. Collecting terms and evaluating at $\hat{z}=0$, we obtain

$$
\begin{aligned}
\frac{1}{\mathrm{Nu}}= & 1-\frac{1}{6} \operatorname{Pe} f(\hat{t})+\mathrm{Pe}^{2}\left\{\frac{3}{40} \mathrm{St} \frac{d f}{d \hat{t}}+\frac{1}{40}[f(\hat{t})]^{2}\right\} \\
& +O\left(\mathrm{Pe}^{3}\right) .
\end{aligned}
$$

The product $\operatorname{Pe} f(\hat{t})=\alpha_{0} f(\omega t) \delta^{2} / \kappa$ can be regarded as a time-dependent Péclet number relevant at each moment in time. Hence, we see that (T7a) can be applied in a quasi-steady fashion for low-frequency flows with weak advection (i.e., flows with $\mathrm{St} \mathrm{Pe} \ll 1$ and $\mathrm{Pe} \ll 1$ ). The first unsteady correction occurs at $O\left(\mathrm{StPe}^{2}\right)$.

\section{b. Linear surface straining model}

The derivation for the linear surface straining model proceeds in the same fashion as that in the previous subsection, except that the nature of the advection term and the lower boundary condition are modified to reflect 
the different assumptions of the two theories, and the algebra becomes a little more involved. From (T1) and (T2b), the heat equation for the linear surface straining model is

$\frac{\partial^{2} \theta}{\partial \hat{z}^{2}}=-\left[\frac{\pi}{2}+\operatorname{Pe} f(\hat{t})\right] \hat{z} \frac{\partial \theta}{\partial \hat{z}}+\operatorname{StPe} \frac{\partial \theta}{\partial \hat{t}}$

and, seeking solutions of the form $\theta=\theta_{0}(\hat{z}, \hat{t})+\operatorname{Pe}_{0} \theta_{1}$ $(\hat{z}, \hat{t})+\mathrm{Pe}_{0}^{2} \theta_{2}(\hat{z}, \hat{t})+\cdots$, the boundary conditions (T3b) and (T4b) yield

$$
\begin{aligned}
& \theta_{0}=\theta_{i}=0 \quad \text { as } \quad \hat{z} \rightarrow-\infty, \quad \text { and } \\
& \frac{\partial \theta_{0}}{\partial \hat{z}}=-1, \\
& \frac{\partial \theta_{i}}{\partial \hat{z}}=0 \quad \text { at } \quad \hat{z}=0, \quad i=1,2, \ldots
\end{aligned}
$$

The leading-order heat equation yields a steady balance between diffusion and advection by the free convective flow

$$
\frac{\partial^{2} \theta_{0}}{\partial \hat{z}^{2}}+\frac{\pi}{2} \hat{z} \frac{\partial \theta_{0}}{\partial \hat{z}}=0
$$

with solution

$$
\theta_{0}=-\operatorname{erfc}\left[-\frac{\hat{z} \sqrt{\pi}}{2}\right]
$$

which is identical to the Leighton et al. (2003) result.

The first-order heat equation at $O(\mathrm{Pe})$ is

$$
\frac{\partial^{2} \theta_{1}}{\partial \hat{z}^{2}}+\frac{\pi}{2} \hat{z} \frac{\partial \theta_{1}}{\partial \hat{z}}=-f(\hat{t}) \hat{z} \frac{\partial \theta_{0}}{\partial \hat{z}}
$$

representing a balance of diffusion and advection of the temperature perturbation $\theta_{1}$ by the free convection flow, and quasi-steady advection of the leading-order temperature $\theta_{0}$ by the imposed upwelling flow (the term on the right hand side). Substituting for $\theta_{0}$ from (A13), this integrates to yield

$\theta_{1}=f(\hat{t}) \frac{1}{\pi}\left\{\operatorname{erfc}\left[-\frac{\hat{z} \sqrt{\pi}}{2}\right]-\hat{z} \exp \left[-\frac{\hat{z}^{2} \pi}{4}\right]\right\}$

so that the first-order perturbation to the temperature is quasi steady.

The unsteady term $\partial \theta / \partial t$ is again first significant at $O\left(\mathrm{Pe}^{2}\right)$, where (A10) yields

$$
\begin{aligned}
\frac{\partial^{2} \theta_{2}}{\partial \hat{z}^{2}}+\frac{\pi}{2} \hat{z} \frac{\partial \theta_{2}}{\partial \hat{z}}= & -[f(\hat{t})]^{2} \frac{\hat{z}^{3}}{2} \exp \left[-\frac{\pi}{4} \hat{z}^{2}\right] \\
& +\operatorname{Stf}^{\prime}(\hat{t}) \frac{1}{\pi}\left\{\operatorname{erfc}\left[-\frac{\hat{z} \sqrt{\pi}}{2}\right]\right. \\
& \left.-\hat{z} \exp \left[-\frac{\hat{z}^{2} \pi}{4}\right]\right\}
\end{aligned}
$$

after eliminating $\theta_{1}$. Liberal use of integration by parts leads to the solution

$$
\begin{aligned}
\theta_{2}= & \frac{3}{2 \pi^{2}}[f(\hat{t})]^{2}\left\{\operatorname{erfc}\left[-\frac{\hat{z} \sqrt{\pi}}{2}\right]-\left(\hat{z}+\frac{\pi}{6} \hat{z}^{3}\right) \exp \left[-\frac{\hat{z}^{2} \pi}{4}\right]\right\}+\operatorname{St} f^{\prime}(\hat{t}) \frac{2}{\pi^{2}}\left\{\hat{z} \exp \left[-\frac{\hat{z}^{2} \pi}{4}\right]-\operatorname{erfc}\left[-\frac{\hat{z} \sqrt{\pi}}{2}\right]\right\} \\
& +\operatorname{Stf}^{\prime}(\hat{t}) \frac{1}{\pi} \int_{-\infty}^{\hat{z}} \int_{0}^{\xi} \int_{-\infty}^{\eta}\left[-\frac{\pi}{4}\left(\xi^{2}+\zeta^{2}-\eta^{2}\right)\right] d \zeta d \eta d \xi .
\end{aligned}
$$

Combining (A13), (A15), and (A17) and evaluating at $\hat{z}=0$, we find a nondimensional bulk-skin temperature difference

$$
\begin{aligned}
\frac{1}{\mathrm{Nu}}= & 1-\frac{1}{\pi} \operatorname{Pe} f(\hat{t})+\frac{3}{2 \pi^{2}}[\operatorname{Pe} f(\hat{t})]^{2} \\
& -\operatorname{StPe}^{2} f^{\prime}(\hat{t}) \frac{2}{\pi^{2}}(1+\log 2)+O\left(\mathrm{Pe}^{3}\right),
\end{aligned}
$$

where the factor $2 \log 2$ was obtained using the commercial software Mathematica to evaluate the definite integral resulting from the final term in (A17). Recalling that $\operatorname{Pe} f(\hat{t})$ is a time-dependent Péclet number, the results (A9) for the Osborne (1965) theory and (A18) for the linear surface straining model both show that the leading-order variation in skin temperature [the $O(\mathrm{Pe})$ term] is quasi steady at leading order for $\mathrm{Pe} \ll 1$ and $\mathrm{StPe} \ll 1$.

\section{APPENDIX B}

\section{Boundary Layer Structure for Large Péclet Numbers}

For large Péclet numbers, we can use the method of matched asymptotic expansions to reveal the structure of the boundary layer relevant to the model of Osborne (1965), by solving the nondimensional heat equation (T1) and boundary conditions (T3a) and (T4a) for 
TABLE C1. Table of experimental conditions and measured strain rate, bulk temperature, heat flux, and skin temperature. Skin temperatures are indicated for the central measurement region $(\mathrm{C})$ and far-field measurement region $(\mathrm{F})$.

\begin{tabular}{|c|c|c|c|c|c|c|c|c|c|}
\hline $\begin{array}{c}\alpha \\
\mathrm{s}^{-1}\end{array}$ & $\begin{array}{l}T_{B} \\
{ }^{\circ} \mathrm{C}\end{array}$ & $\begin{array}{c}Q \\
\mathrm{~W} \mathrm{~m}^{-2}\end{array}$ & $\begin{array}{c}T_{S}(\mathrm{C}) \\
{ }^{\circ} \mathrm{C}\end{array}$ & $\begin{array}{c}T_{S}(\mathrm{~F}) \\
{ }^{\circ} \mathrm{C}\end{array}$ & $\begin{array}{c}\alpha \\
\mathrm{s}^{-1}\end{array}$ & $\begin{array}{l}T_{B} \\
{ }^{\circ} \mathrm{C}\end{array}$ & $\begin{array}{c}Q \\
\mathrm{~W} \mathrm{~m}^{-2}\end{array}$ & $\begin{array}{c}T_{S}(\mathrm{C}) \\
{ }^{\circ} \mathrm{C}\end{array}$ & $\begin{array}{c}T_{S}(\mathrm{~F}) \\
{ }^{\circ} \mathrm{C}\end{array}$ \\
\hline 0.000 & 25.57 & 198.3 & 24.63 & 24.71 & 0.057 & 21.96 & 82.9 & 21.23 & 21.37 \\
\hline 0.000 & 25.52 & 204.9 & 24.62 & 24.49 & 0.057 & 21.93 & 78.7 & 21.32 & 21.21 \\
\hline 0.000 & 25.44 & 203.7 & 24.41 & 24.39 & 0.057 & 21.90 & 80.6 & 21.24 & 21.15 \\
\hline 0.000 & 25.38 & 198.7 & 24.45 & 24.49 & 0.057 & 21.60 & 50.1 & 20.75 & 20.83 \\
\hline 0.000 & 25.33 & 197.6 & 24.21 & 24.30 & 0.057 & 21.59 & 86.5 & 20.72 & 20.77 \\
\hline 0.000 & 23.68 & 137.0 & 22.61 & 22.63 & 0.057 & 21.58 & 89.0 & 20.70 & 20.77 \\
\hline 0.000 & 23.66 & 176.0 & 22.69 & 22.74 & 0.057 & 21.54 & 84.4 & 20.73 & 20.72 \\
\hline 0.000 & 22.45 & 86.3 & 21.98 & 21.93 & 0.057 & 21.52 & 84.3 & 20.63 & 20.65 \\
\hline 0.000 & 22.40 & 84.4 & 21.82 & 21.81 & 0.057 & 18.90 & 13.9 & 18.64 & 18.37 \\
\hline 0.000 & 22.20 & 81.6 & 21.58 & 21.64 & 0.057 & 18.89 & 15.5 & 18.54 & 18.39 \\
\hline 0.000 & 22.19 & 79.1 & 21.51 & 21.54 & 0.057 & 18.88 & 14.2 & 18.52 & 18.32 \\
\hline 0.000 & 22.17 & 80.6 & 21.63 & 21.58 & 0.057 & 18.88 & 10.4 & 18.49 & 18.36 \\
\hline 0.000 & 18.92 & 14.8 & 18.42 & 18.46 & 0.057 & 18.87 & 13.6 & 18.54 & 18.37 \\
\hline 0.000 & 18.92 & 25.5 & 18.42 & 18.46 & 0.118 & 23.57 & 119.7 & 22.92 & 22.84 \\
\hline 0.000 & 18.92 & 14.4 & 18.39 & 18.47 & 0.118 & 23.54 & 119.7 & 22.59 & 22.72 \\
\hline 0.000 & 18.91 & 16.5 & 18.35 & 18.41 & 0.118 & 23.51 & 122.0 & 22.80 & 22.82 \\
\hline 0.043 & 22.20 & 91.7 & 21.39 & 21.37 & 0.118 & 23.44 & 124.7 & 22.58 & 22.69 \\
\hline 0.043 & 22.19 & 91.4 & 21.47 & 21.31 & 0.118 & 23.41 & 125.7 & 22.62 & 22.56 \\
\hline 0.043 & 22.18 & 90.0 & 21.37 & 21.50 & 0.118 & 21.54 & 68.9 & 20.88 & 20.82 \\
\hline 0.043 & 22.15 & 88.2 & 21.43 & 21.40 & 0.118 & 21.53 & 70.4 & 20.94 & 20.83 \\
\hline 0.043 & 22.14 & 87.9 & 21.17 & 21.21 & 0.118 & 21.50 & 72.2 & 20.97 & 20.79 \\
\hline 0.057 & 24.66 & 181.9 & 23.49 & 23.56 & 0.118 & 21.45 & 71.8 & 20.92 & 20.76 \\
\hline 0.057 & 24.62 & 180.3 & 23.67 & 23.66 & 0.118 & 21.43 & 74.5 & 20.89 & 20.74 \\
\hline 0.057 & 24.60 & 180.8 & 23.42 & 23.41 & 0.158 & 23.85 & 114.4 & 23.31 & 23.05 \\
\hline 0.057 & 24.57 & 180.7 & 23.43 & 23.53 & 0.158 & 23.82 & 110.1 & 23.35 & 23.03 \\
\hline 0.057 & 24.52 & 181.0 & 23.46 & 23.51 & 0.158 & 23.79 & 111.2 & 23.35 & 23.03 \\
\hline 0.057 & 23.18 & 102.3 & 22.41 & 22.43 & 0.158 & 23.76 & 110.3 & 23.24 & 23.04 \\
\hline 0.057 & 23.17 & 99.4 & 22.43 & 22.43 & 0.426 & 23.29 & 133.2 & 22.82 & 22.38 \\
\hline 0.057 & 23.14 & 96.9 & 22.47 & 22.41 & 0.426 & 23.26 & 136.0 & 22.74 & 22.36 \\
\hline 0.057 & 23.11 & 92.6 & 22.36 & 22.33 & 0.426 & 23.24 & 132.9 & 22.64 & 22.18 \\
\hline 0.057 & 23.09 & 92.8 & 22.32 & 22.33 & 0.426 & 23.22 & 129.8 & 22.45 & 22.04 \\
\hline 0.057 & 21.96 & 77.2 & 21.26 & 21.23 & 0.426 & 23.20 & 130.4 & 22.69 & 22.07 \\
\hline
\end{tabular}

steady flow $(\partial / \partial t \equiv 0)$. The limit $\mathrm{Pe} \rightarrow \infty$ gives a singular perturbation to (T1), thus we need to resolve an additional boundary layer within the existing skin layer. The solution far from the interface is obtained by letting $\mathrm{Pe} \rightarrow \infty$ in (T1), which yields a limit that is dominated by advection,

$$
-\hat{z} \frac{\partial \theta}{\partial \hat{z}}=0
$$

with the general solution $\theta=$ constant. Matching to the bulk temperature at the base of the skin layer $(\theta=0$ at $\hat{z}=-1$ ), we obtain the outer solution

$$
\theta=0
$$

so that the temperature remains at the bulk temperature at leading order throughout the lower part of the skin layer. This solution is unable to satisfy the constant heat flux boundary condition at the free surface, thus we must rescale to resolve a boundary layer in a narrow region near to the interface. Physically, diffusion must become important in a region near to the interface, and the rescaling determines the depth of the region with a balance between advection and diffusion.

To retain both the advection and diffusion terms in (T1) and to also satisfy the boundary condition of constant heat flux at the free surface $(\partial \theta / \partial \hat{z}=-1$ at $\hat{z}=0)$, we use the rescaled coordinate $\zeta=\mathrm{Pe}^{1 / 2} \hat{z}$ and a rescaled temperature $\phi=\mathrm{Pe}^{1 / 2} \theta$. The heat equation (T1) then becomes

$$
-\zeta \frac{\partial \phi}{\partial \zeta}=\frac{\partial^{2} \phi}{\partial \zeta^{2}}
$$

with free surface boundary condition

$$
\frac{\partial \phi}{\partial \zeta}=-1 \quad \text { at } \quad \zeta=0 .
$$


The second boundary condition comes from the matching of the inner and outer solutions, requiring

$$
\phi \rightarrow 0 \text { as } \zeta \rightarrow \infty \text {. }
$$

The solution to (B3)-(B5) is

$$
\phi(\zeta)=-\sqrt{\frac{\pi}{2}} \operatorname{erfc}\left[\frac{\zeta}{\sqrt{2}}\right]
$$

which is asymptotically equivalent to (12) as $\mathrm{Pe} \rightarrow \infty$. We see immediately from this asymptotic solution that the leading-order temperature variation is confined entirely to the near-surface region, where $\zeta=O(1)$ and hence $\hat{z}=O\left(\mathrm{Pe}^{-1 / 2}\right)$. In dimensional terms, this means the thermal boundary layer thickness adjusts to a new scale of $z=O(\sqrt{\kappa / \alpha})$.

\section{APPENDIX C}

\section{Table of Experimental Conditions and Measurements}

Table $\mathrm{C} 1$ provides a comprehensive list of conditions for each experimental run.

\section{REFERENCES}

Castro, S. L., G. A. Wick, and W. J. Emery, 2003: Further refinements to models for the bulk-skin sea surface temperature difference. J. Geophys. Res., 108, 3377, doi:10.1029/2002JC001641.

Chapman, C. J., and M. R. E. Proctor, 1980: Nonlinear RayleighBénard convection between poorly conducting boundaries. J. Fluid Mech., 101, 759-782.

Csanady, G. T., 1990: The role of breaking wavelets in air-sea gas transfer. J. Geophys. Res., 95, 749-759.

Ewing, G. C., and E. D. McAlister, 1960: On the thermal boundary layer of the ocean. Science, 131, 1374-1376.

Fairall, C. W., E. F. Bradley, D. P. Rogers, J. B. Edson, and G. S. Young, 1996: Bulk parameterization of air-sea fluxes for Tropical Ocean-Global Atmosphere Coupled OceanAtmosphere Response Experiment. J. Geophys. Res., 101, 3747-3764.

Farrar, J. T., C. J. Zappa, R. A. Weller, and A. T. Jessup, 2007: Sea surface temperature signatures of oceanic internal waves in low winds. J. Geophys. Res., 112, C06014, doi:10.1029/2006JC003947.

Fortescue, G. E., and J. R. A. Pearson, 1967: On gas absorption into a turbulent liquid. Chem. Eng. Sci., 33, 1163-1176.

Gasparovic, R. F., J. R. Apel, and E. S. Kasischke, 1988: An overview of the SAR internal wave signature experiment. J. Geophys. Res., 93 (C10), 12 304-12 316.

Handler, R. A., R. I. Leighton, G. B. Smith, and R. Nagaosa, 2003: Surfactant effects on passive scalar transport in a fully developed turbulent flow. Int. J. Heat Mass Transfer, 46, 2219-2238.

Howard, L. N., 1966: Convection at high Rayleigh number. Proceedings of the Eleventh International Congress of Applied Mechanics, H. Görtler, Ed., Springer-Verlag, 1109-1115.
Jähne, B., and H. Haußecker, 1998: Air-water gas exchange. Annu. Rev. Fluid Mech., 30, 443-468, doi:10.1146/annurev.fluid.30.1.443.

_ K. O. Münnich, and U. Siegenthaler, 1979: Measurements of gas exchange and momentum transfer in a circular wind-water tunnel. Tellus, 31, 321-328.

Jarvis, N. L., 1962: The effect of monomolecular films on surface temperature and convective motion at the water/air interface. Naval Research Lab Tech. Rep. AD0270608, 13 pp.

Jessup, A. T., and V. Hesany, 1996: Modulation of ocean skin temperature by swell waves. J. Geophys. Res., 101, 6501-6511.

, C. J. Zappa, and H. Yeh, 1997: Defining and quantifying microscale wave breaking with infrared imagery. J. Geophys. Res., 102, 23 145-23 154.

Katsaros, K. B., W. T. Liu, J. A. Businger, and J. E. Tillman, 1977: Heat transport and thermal structure in the interfacial boundary layer measured in an open tank of water in turbulent free convection. J. Fluid Mech., 83, 311-335.

Leighton, R. I., G. B. Smith, and R. A. Handler, 2003: Direct numerical simulations of free convection beneath an air-water interface at low Rayleigh numbers. Phys. Fluids, 15, 31813193.

Marmorino, G. O., and G. B. Smith, 2005: Bright and dark ocean whitecaps observed in the infrared. Geophys. Res. Lett., 32, L11604, doi:10.1029/2005GL023176.

—_ _ , and G. J. Lindemann, 2004: Infrared imagery of ocean internal waves. Geophys. Res. Lett., 31, L11309, doi:10.1029/ 2004GL020152.

- — , J. V. Toporkov, M. A. Sletten, D. Perkovic, and S. Frasier, 2008: Evolution of ocean slicks under a rising wind. Geophys. Res. Lett., 113, C04030, doi:10.1029/2007JC004538.

McKenna, S. P., and W. R. McGillis, 2004: The role of free-surface turbulence and surfactants in air-water gas transfer. Int. J. Heat Mass Transfer, 47, 539-553.

Okuda, K., 1983: Internal flow structure of short wind waves. J. Oceanogr. Soc. Japan, 38, 28-42.

Osborne, M. F. M., 1965: The effect of convergent and divergent flow patterns on infrared and optical radiation from the sea. Ocean Dyn., 18, 1-25.

Pearson, J. R. A., 1958: On the convection cells induced by surface tension. J. Fluid Mech., 4, 489-500.

Peirson, W. L., and M. L. Banner, 2003: Aqueous surface layer flows induced by microscale breaking wind waves. J. Fluid Mech., 479, 1-38.

Saunders, P., 1967: The temperature at the ocean-air interface. J. Atmos. Sci., 24, 269-273.

Saylor, J. R., G. B. Smith, and K. A. Flack, 2000: The effect of a surfactant monolayer on the temperature field of a water surface undergoing evaporation. Int. J. Heat Mass Transfer, 43, 3073-3086.

Smith, G. B., R. J. Volino, R. A. Handler, and R. I. Leighton, 2001: The thermal signature of a vortex pair impacting a free surface. J. Fluid Mech., 444, 49-78.

Soloviev, A. V., and P. Schlüssel, 1994: Parameterization of the cool skin of the ocean and of the air-ocean gas transfer on the basis of modeling surface renewal. J. Phys. Oceanogr., 24, 1339-1346.

Turney, D. E., W. C. Smith, and S. Banerjee, 2005: A measure of near-surface fluid motions that predicts air-water gas transfer in a wide range of conditions. Geophys. Res. Lett., 32, L04067, doi:10.1029/2004GL021671.

Walsh, E. J., R. Pinkel, D. E. Hagan, R. A. Weller, C. W. Fairall, D. P. Rodgers, S. P. Burns, and M. Baumgartner, 1998: Coupling of internal waves on the main thermocline to the diurnal 
surface layer and sea surface temperature during the Tropical Ocean-Global Atmosphere Coupled Ocean-Atmosphere Response Experiment. J. Geophys. Res., 103, 12 613-12 628.

Wick, G. A., W. J. Emery, L. H. Kantha, and P. Schlüssel, 1996: The behavior of the bulk-skin sea surface temperature difference under varying wind speed and heat flux. J. Phys. Oceanogr., 26, 1969-1987.

J. C. Ohlmann, C. W. Fairall, and A. T. Jessup, 2005: Improved oceanic cool-skin corrections using a refined solar penetration model. J. Phys. Oceanogr., 35, 1986-1996.
Woodcock, A. H., and H. Stommel, 1947: Temperatures observed near the surface of a fresh-water pond at night. J. Meteor., 4, 102-103.

Zappa, C. J., and A. T. Jessup, 2005: High-resolution airborne infrared measurements of ocean skin temperature. IEEE Geosci. Remote Sens. Lett., 2, 146-150.

— W. W. Asher, A. T. Jessup, J. Klinke, and S. R. Long, 2004: Microbreaking and the enhancement of air-water transfer velocity. J. Geophys. Res., 109, C08S16, doi:10.1029/ $2003 J C 001897$. 